\title{
lonized gas kinematics and massive star formation in NGC 1530
}

\author{
A. Zurita ${ }^{1}$, M. Relaño ${ }^{2}$, J. E. Beckman ${ }^{2,3}$, and J. H. Knapen ${ }^{4}$ \\ ${ }^{1}$ Isaac Newton Group of Telescopes, Apartado de Correos 321, 38700, Santa Cruz de La Palma, Canarias, Spain \\ e-mail: azurita@ing.iac.es \\ 2 Instituto de Astrofísica de Canarias, c. Vía Láctea s/n, 38200, La Laguna, Tenerife, Spain \\ e-mail: mpastor@1l.iac.es \\ ${ }^{3}$ Consejo Superior de Investigaciones Científicas (CSIC), Spain \\ e-mail: jeb@ll.iac.es \\ ${ }^{4}$ University of Hertfordshire, Hatfield, Herts, AL10 9AB, UK \\ e-mail: knapen@star.herts.ac.uk
}

Received 10 June 2003 / Accepted 7 July 2003

\begin{abstract}
We present emission line mapping of the strongly barred galaxy NGC 1530 obtained using Fabry-Pérot interferometry in $\mathrm{H} \alpha$, at significantly enhanced angular resolution compared with previously published studies. The main point of the work is to examine in detail the non-circular components of the velocity field of the gas, presumably induced by the strongly non-axisymmetric gravitational potential of the bar. To do this we first derive a model rotation curve making minimum assumptions about kinematic symmetry, and go on to measure the non-circular component of the full radial velocity field. This clearly reveals the streaming motions associated with the spiral density wave producing the arms, and the quasi-elliptical motions with speeds of order $100 \mathrm{~km} \mathrm{~s}^{-1}$ aligned with the bar. It also shows in some detail how these flows swing in towards and around the nucleus as they cross a circumnuclear resonance, from the dominant " $x_{1}$ orbits" outside the resonance to " $x_{2}$ orbits" within it. Comparing cross-sections of this residual velocity map along and across the bar with the surface brightness map in $\mathrm{H} \alpha$ indicates a systematic offset between regions of high non-circular velocity and massive star formation. To investigate further we produce maps of velocity gradient along and across the bar. These illustrate very nicely the shear compression of the gas, revealed by the location of the dust lanes along loci of maximum velocity gradient perpendicular to the bar. They also show clearly how shear, seen in our data as velocity gradient perpendicular to the flow, acts to inhibit massive star formation, whereas shocks, seen as strong velocity gradients along the flow vector, act to enhance it. Although the inhibiting effect of gas shear flow on star formation has long been predicted, this is the clearest observational illustration so far of the effect, thanks to the strong shock-induced counterflow system in the bar. It is also the clearest evidence that dust picks out shock-induced inflow along bars. These observations should be of considerable interest to those modelling massive star formation in general.
\end{abstract}

Key words. galaxies: general - galaxies: individual: NGC 1530 - galaxies: ISM - galaxies: spiral galaxies: kinematics and dynamics - ISM: kinematics and dynamics

\section{Introduction}

The interactive relation between dynamics and morphology is a well known aspect of barred galaxies. It has been invoked to throw light on one of the more interesting questions in galactic evolution: by what mechanism or mechanisms is material driven in to regions close to the nucleus where it can participate in star formation, or contribute to the mass of the central supermassive black hole, and to the activity around it (Shlosman et al. 1989). As an archetypal barred galaxy NGC 1530 has received considerable attention both morphologically and kinematically. These studies have been facilitated observationally by the strength and length of the main bar, which compensate for its distance. The latter is 36.6 Mpc (Tully \& Fisher 1988), which agrees with a more recent calculation of $37 \mathrm{Mpc}$ using

Send offprint requests to: J. E. Beckman, e-mail: jeb@1l.iac.es its recession velocity of $2666 \mathrm{~km} \mathrm{~s}^{-1}$ (Young et al. 1989) and a value for $H_{0}$ of $72 \mathrm{~km} \mathrm{~s}^{-1} \mathrm{Mpc}^{-1}$ (Freedman et al. 2001). In spite of this, the bar and its accompanying kinematics can be well observed because its deprojected radial length is some $14 \mathrm{kpc}$. The strength of the bar (it has one of the highest bar torques measured by Buta et al. 2003) has attracted the attention of observers to the distinctive phenomenology of this galaxy. In recent years two groups have made major contributions to the study of NGC 1530 which are particularly relevant to the work presented here. They are Regan et al. (1995, 1996, 1997) and the group of Downes \& Reynaud (Downes et al. 1996; Reynaud \& Downes 1997, 1998, 1999; Greve et al. 1999). The types of observations these groups present overlap to some degree. The group of Downes \& Reynaud base most of their work on velocity and surface luminosity mapping in $\mathrm{CO}$ with some additional stellar information presented in 
Greve et al. (1999), while Regan et al.'s most important contributions come from their velocity and surface brightness mapping in $\mathrm{H} \alpha$ with a useful contribution from $\mathrm{CO}$ supplemented by near-IR mapping in the initial paper of their published series.

NGC 1530 is a dramatic twin-armed spiral galaxy, with arms breaking away sharply from the ends of the long primary bar. It is notable that these arms, though trailing, have shorter opposing lengths, curving inward from the tips of the bar as prolongations of the main arms. They are well brought out in $\mathrm{H} \alpha$ as seen in Regan et al. (1995) or in Fig. 1a of the present paper. The bar imaged in broad band is wide and contains prominent dust lanes which, as the publications by Regan et al. and by Downes \& Reynaud listed above well point out, pick out shocked gas following trajectories generally aligned with the bar. These are almost straight along the bar but begin to curve inwards from a galactocentric distance of some $2 \mathrm{kpc}$, bending sharply in towards the nucleus. They link the prominent star-forming regions at the ends of the bar, seen very well here in $\mathrm{H} \alpha$ in Fig. 1a, with the even more prominent star-forming ring feature with radius $\sim 2 \mathrm{kpc}$ noted in blue images by Buta (1984) and by Wray (1988) and also clearly picked out in $\mathrm{H} \alpha$ in Fig. 1a. Combining near-IR with optical data to give colour maps, Regan et al. (1995) explored the stellar and dust morphology in the bar and in the central zone following the lanes right into the nucleus. One of the aims of the present paper is to elucidate further the relation between gas flows and star formation which are implied in these morphological data described above.

However, this aim has been pursued with considerable achievement previously by the two groups mentioned above. Regan et al. (1996) combined atomic hydrogen $21 \mathrm{~cm}$ measurements with ionized hydrogen $\mathrm{H} \alpha$ Fabry-Pérot spectroscopy to derive a rotation curve for NGC 1530 . From this they derived a bar pattern speed of $20 \mathrm{~km} \mathrm{~s}^{-1} \mathrm{kpc}^{-1}$ and used it to predict resonances at radii coinciding with prominent morphological features, including an outer gaseous ring seen in $\mathrm{HI}$, an inner ring seen in $\mathrm{H} \mathrm{I}$ and $\mathrm{H} \alpha$, and the nuclear star-forming ring mentioned above. Downes et al. (1996) mapped the galaxy in CO and identified the major concentrations of potentially star forming molecular gas; these are located over the central prominent inner disc zone of radius $\sim 2 \mathrm{kpc}$ and extend out more weakly over the bar to less prominent concentrations over the ends of the bar. They added interferometric mapping of the central zone, finding a circumnuclear ring structure with gas in orbits apparently perpendicular to the elongated orbits in the outer parts of the bar. They relate these patterns to the predictions of resonance models (Athanassoula 1984; Jenkins \& Binney 1994) in which gas within corotation moves along $x_{1}$ orbits along the bar until it reaches an inner Lindblad resonance (ILR) where it moves into the $x_{2}$ orbits perpendicular to the bar. A neat schematic diagram in Fig. 10 of Downes et al. (1996) illustrates the predicted behaviour of the gas under these circumstances. Although the stars follow quasi-elliptical $x_{1}$ orbits, in the case of gas, the shocks are produced along the bar and specially at the ends of the bar, where orbit crowding occurs, as was well predicted in Roberts et al. (1979). However, as the gas reaches the outer limit of the zone dominated by $x_{2}$ dynamics, it suffers another shock, and is pulled through a right-angle, falling in again towards the nucleus. Gas on $x_{1}$ orbits with an impact parameter a little larger with respect to the nucleus is less affected by the effects of the resonance, and proceeds along the outgoing edge of the bar, albeit with declining velocities. It is just this kind of arrangement for allowing gas to fall to the centres of barred galaxies which was invoked by Shlosman et al. (1989) in their "bars within bars" scenario as a fueling mechanism for the different types of circumnuclear activity: starbursts and AGN.

Using Fabry-Pérot H $\alpha$ mapping of NGC 1530, Regan et al. (1997) demonstrated that the two-dimensional $\mathrm{H} \alpha$ velocity field in the bar region shows the skew pattern predicted for $x_{1}$ orbits superposed on the general galactic rotation. They went on to combine hydrodynamical models with their observations to compute a net inflow rate to the nuclear zone of $\sim 1 M_{\odot}$ per year, with the remaining gas spraying back outwards along the major bar axis before coupling into a new inflow cycle. Using molecular emission from $\mathrm{CO}$ and $\mathrm{HCN}$, the former at angular resolution of $1.8^{\prime \prime}$ and the latter at 3.6", Reynaud \& Downes (1997) detected steep velocity gradients spatially confined to two arcs coupling into a ring. These arcs represent shock fronts where the inflow along the bar is being constrained to change direction and assume the $x_{2}$ orbital configuration along the nucleus. These arcs correspond to two small arm features picked out in $\mathrm{H} \alpha$ to the north and south of the circumnuclear zone of enhanced star formation (see Fig. 1a of the present paper). The points where the arcs couple to the circumnuclear ring are the main emitters in $\mathrm{HCN}$, suggesting points of maximum molecular density. Reynaud \& Downes postulate the presence of two ILR's: one at $1.2 \mathrm{kpc}$ from the nucleus where the shock arcs are rooted, and one very close to the nucleus, at the inner edge of the molecular ring. They support these inferences using resonance theory and their rotation curve, which is derived kinematically but supported by a dynamical model. In a subsequent article Reynaud \& Downes (1999) map the same area in two emission lines each of ${ }^{12} \mathrm{CO}$ and ${ }^{13} \mathrm{CO}$, showing that the molecular gas density is highest in the circumnuclear ring and not so high in the arcs referred to above.

Perhaps of most interest and relevance for the study we present here is that of Reynaud \& Downes (1998), who use their CO kinematics of the whole bar region in NGC 1530 to explore the relation between velocity changes and star formation. They conclude that strong velocity changes, which represent shocks and shear, are negatively correlated with star formation and that the distribution of star formation does not correspond fully to the distribution of available molecular gas, as traced by $\mathrm{CO}$. Although it is true that the major concentration is found in the central circumnuclear zone where star formation is most enhanced, there is considerable CO measured along the bar as well as at its ends, while the star formation is clearly weak along of the bar. Reynaud \& Downes find velocity jumps across the dust lanes from velocities of order $100 \mathrm{~km} \mathrm{~s}^{-1}$ along the bar in one direction, to similar velocities in the reverse direction. These jumps maximize half way out along the bar from the nucleus on both sides. They conclude that steep velocity gradients, both shear and shock, prevent molecular clouds from condensing to form stars. One of the main aims 
of the present paper is to examine this hypothesis in a little more detail using an $\mathrm{H} \alpha$ velocity field.

We present new high resolution velocity and intensity maps of NGC 1530, obtained in $\mathrm{H} \alpha$ emission using a Fabry-Pérot interferometer. The instrument provides a three-dimensional "data cube", akin to a conventional radiastronomical set of line maps, in which the emission intensity is given as a function of three coordinates: two positional coordinates and the velocity. From this "data cube" one goes on to compute maps in integrated line intensity, line peak velocity, and velocity dispersion. The next step is to obtain the rotation curve of the galaxy and to subtract it off two-dimensionally from the complete observed velocity field. This allows us to obtain the non-circular motions due to non-axisymmetric elements of the galaxy. For NGC 1530 the dominant source of non-axisymmetry is the strong bar of length almost $28 \mathrm{kpc}$, which dominates the appearance of the galactic disk. We also find kinematic signatures of the streaming motions in the sharply defined spiral arms. Besides we also see, in the central $5 \mathrm{kpc}$, evidence for a distinct kinematic structure predicted by classical resonance theory, and already carefully observed and characterised in the studies by Regan and coworkers, and by Reynaud \& Downes in the references given above.

As a further step we have prepared plots of the velocity gradients in the whole of the bar and arm structure as imaged in $\mathrm{H} \alpha$ emission. We have related the velocity gradient morphology to the distribution of the massive star formation in arms, bar, and the central zone. Our main steps forward compared with previous studies are due to the uniformly high angular resolution we could obtain over the full image. This has enabled us to make a more precise definition of the rotation curve, and hence an improved map of the non-circular velocity field, both around the main bar, and in the central zone. From the two-dimensional display of the non-circular velocities we have gone on to derive a map of the velocity gradients, with a resolution better than $2^{\prime \prime}$ over the whole field of the galaxy. Such a map has not been previously produced, so we are able to make progress here in characterising the geometrical and physical relationships between velocity gradients and star formation.

\section{Observations and data reduction}

The Fabry-Pérot observations of NGC 1530 were taken at the Roque de los Muchachos Observatory with the $4.2 \mathrm{~m}$ William Herschel Telescope (WHT) on the night of September 2nd, 1998. The TAURUS-II instrument, equipped with a TEK CCD camera was used to observe the galaxy in the $\mathrm{H} \alpha$ emission line, with a plate scale of $0.292^{\prime \prime}$ per pixel, which corresponds to a pixel scale $179 \mathrm{pc} \operatorname{arcsec}^{-1}$ at the distance of the galaxy $(37 \mathrm{Mpc})$. The observations consisted of exposures of $150 \mathrm{~s}$ at equally spaced positions of the etalon, at intervals of $0.407 \AA$, corresponding to $18.62 \mathrm{~km} \mathrm{~s}^{-1}$. The total number of steps was 55 , allowing us to scan the full wavelength range of the $\mathrm{H} \alpha$ line in the galaxy. We used the appropriately redshifted narrow band $\mathrm{H} \alpha$ filter as an order-sorting filter $\left(\lambda_{\mathrm{c}}=6613 \AA\right.$, $\Delta \lambda=15 \AA$, corresponding to the galaxy's systemic velocity $v_{\text {sys }}=2461 \mathrm{~km} \mathrm{~s}^{-1}$; de Vaucouleurs et al. 1991; hereinafter RC3). Wavelength and phase calibration were performed using observations of a calibration lamp (taken before and after the observations of the galaxy), using the TAUCAL package in the FIGARO environment. The spatial resolution of the resulting data set is $\sim 1^{\prime \prime}$. The data set was placed on a correctly oriented spatial grid by comparing positions of point-like features in the $\mathrm{H} \alpha$ Fabry-Pérot intensity map with those in the narrow-band $\mathrm{H} \alpha$ image from Relaño et al. (2003b), yielding maximum uncertainties in positions of $0.3^{\prime \prime}$. The final calibrated data cube has $941 \times 941$ pixels $\times 55$ "planes" in wavelength, separated by $18.62 \mathrm{~km} \mathrm{~s}^{-1}$. We examined plane by plane the data cube and found that it had a considerable number of cosmic ray events. We eliminated them with the task imedit in IRAF, which allowed us to replace the anomalous pixels by a mean background value. We determined which channels of the data set were free of $\mathrm{H} \alpha$ line emission after smoothing the data to a resolution of $2.5^{\prime \prime} \times 2.5^{\prime \prime}$. We found 10 channels free of line emission on the low-velocity side, and 13 channels on the highvelocity side. The continuum was determined by fitting a linear relation to the line-free channels, and was then subtracted from the line emission channels.

In order to study the kinematics on different scales, the data-cube was convolved down from the original resolution of $1^{\prime \prime} \times 1^{\prime \prime}$ to resolutions of $2^{\prime \prime} \times 2^{\prime \prime}, 3^{\prime \prime} \times 3^{\prime \prime}, 6^{\prime \prime} \times 6^{\prime \prime}$ and $10^{\prime \prime} \times 10^{\prime \prime}$. For each data set we obtained the total H $\alpha$ intensity (zeroth moment), velocity (first moment) and velocity dispersion (second moment) maps. The procedure to obtain the moment maps is equivalent for each smoothed data cube so we describe the procedure followed for the full resolution data set only. A reader wanting a fuller description of the procedure can find it in Knapen (1998). The points with low signal were eliminated producing a conditionally transferred data cube, in which values were retained only at positions where the intensity in the smoothed data cube of $3^{\prime \prime} \times 3^{\prime \prime}$ was larger than 2.5 times the rms noise of the smoothed cube; pixel values at all other positions were set to undefined. We then removed noise peaks outside the area where $\mathrm{H} \alpha$ emission is expected by setting the pixel values there to undefined too. This was done interactively by inspecting the high resolution channel maps one by one and comparing them with the corresponding and adjacent channel maps in the $3^{\prime \prime} \times 3^{\prime \prime}$ data set. The resulting data cube was then used to calculate the moment maps using the MOMENTS task in the GIPSY package.

MOMENTS obtains the zeroth, first and second moments of the emission at each spatial pixel in the galaxy and produces a corresponding map for each moment. In the procedure, MOMENTS takes into account the signal only when two conditions are met: the signal is higher than 2.5 times the rms noise of the high-resolution cube, in at least three adjacent channels. The intensity map for the $2^{\prime \prime}$ resolution data cube is shown in Fig. 1a and the first moment (velocity) map is shown in Fig. 1b.

Although the moments procedure is a more useful technique for studying the global kinematics in the galaxy, it must be treated with caution when it is used for a detailed description of the kinematics of individual H II regions. The moments procedure gives an underestimate of the velocity dispersion of the profile (van der Kruit \& Shostak 1982), thus, if a precision measurement of this quantity is required, a better fit to the line profiles, using Gaussian functions, is needed. 

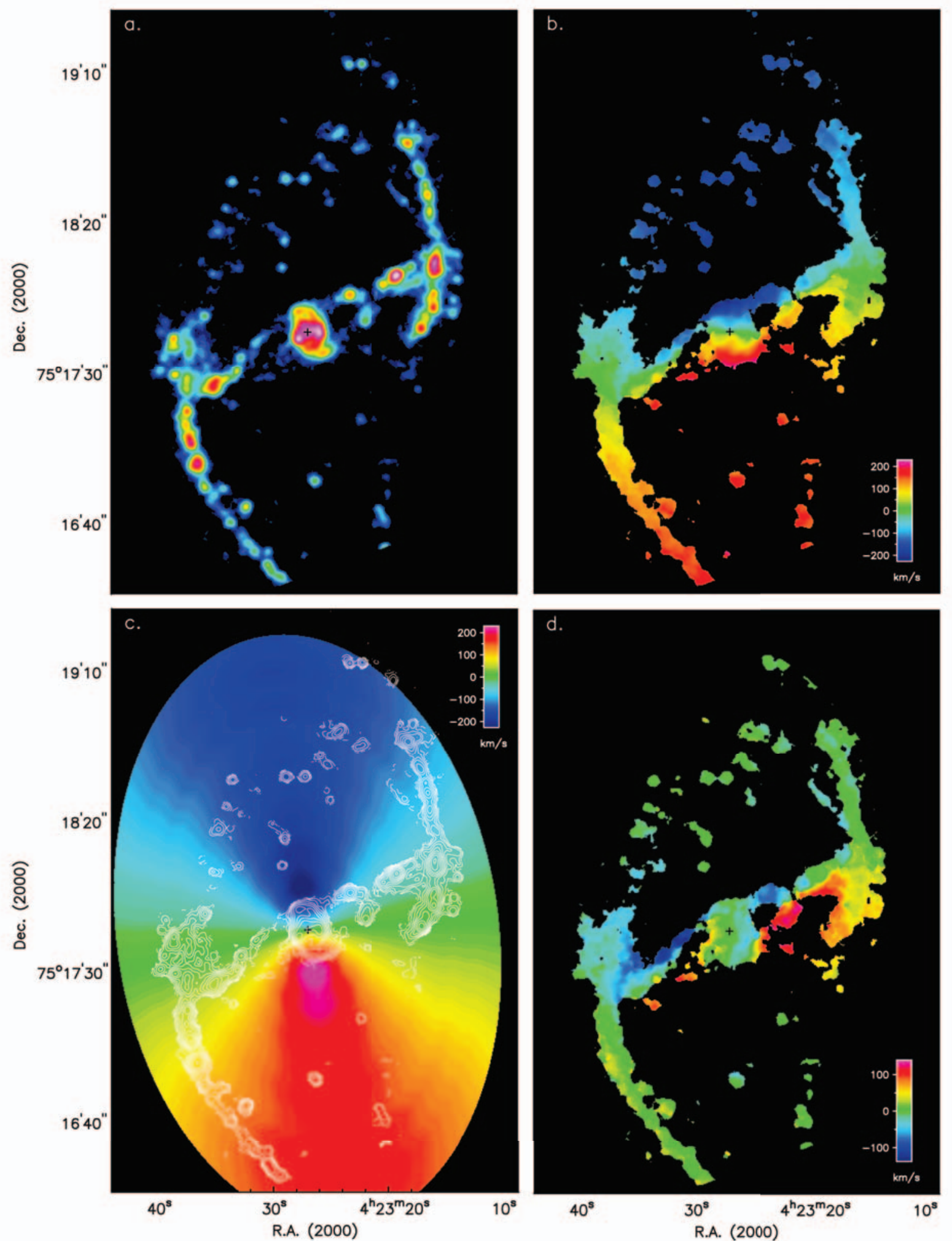

Fig. 1. a) Intensity map (zeroth order moment) of the $\mathrm{H} \alpha$ emission in NGC 1530 . The map was obtained from the $2^{\prime \prime}$ resolution data cube. The kinematic centre is marked with a black cross. b) Velocity map (first moment) of the H $\alpha$ emission in NGC 1530, derived from the $2^{\prime \prime}$ resolution data cube. c) Model velocity map of NGC 1530, obtained from the $\mathrm{H} \alpha$ rotation curve of the galaxy shown in Fig. 3, with H $\alpha$ isointensity contours overlaid. We can clearly see "wiggles" at the radii of the spiral arms, due to the density wave streaming motions not subtracted off from the rotation curve. d) Map of the residual velocities of NGC 1530, obtained by subtracting the model velocity field (Fig. 1c, after taking out the ripples due to the streaming motions in the spiral arms, see text for details) from the velocity map (Fig. 1b). 


\section{Kinematics}

\subsection{Rotation curve}

The rotation curve of the galaxy can be derived from the velocity map, which gives the projected velocity along the line of sight in each position of the galaxy. Assuming that the measurements refer to a position on a single inclined plane, the projected velocity can be related to the rotation velocity in the plane of the galaxy by this expression:

$v(x, y)=v_{\text {sys }}+v_{\text {rot }}(r) \cos (\theta) \sin (i)$

where $v(x, y)$ denotes the radial velocity at the sky coordinates $x$ and $y, v_{\text {sys }}$ the systemic velocity, $v_{\text {rot }}$ the rotational velocity at galactocentric radius $r, i$ the inclination angle of the normal to the galaxy plane to the line of sight, and $\theta$ the azimuthal distance from the major axis in the plane of the galaxy, related to the rotation centre $\left(x_{0}, y_{0}\right)$ and the position angle of the galaxy, PA. We derived the rotation curve following the procedure described by Begeman (1989), where the rotation curve is determined by fitting tilted rings to the observed velocity field. For each ring independently, the parameters $v_{\text {sys }}, v_{\text {rot }}, i$ and $x_{0}, y_{0}$ and PA are obtained making a least-squares fit to Eq. (1) using the observed velocity field $v(x, y)$.

We discarded the points within a certain free-angle $\theta_{\max }$ around the minor axis since they carry less information about the underlying circular velocity of the galaxy and the values obtained in this zone are less accurate due to projection effects. In the case of NGC 1530 a value of $\theta_{\max }=45^{\circ}$ was used to avoid the non-circular motions along the bar. Besides, we give more weight to positions close to the major axis using a weighting function proportional to $|\cos \theta|$ in the fit. The procedure used to obtain each of the parameters which characterise the rotation curve is the following.

The parameters $v_{\mathrm{sys}}, x_{0}$ and $y_{0}$ depend on the symmetry of the velocity field and are the same for each ring. We obtained the position of the kinematic centre $\left(x_{0}, y_{0}\right)$ using the velocity map of $6^{\prime \prime}$ resolution. We took annuli of $3^{\prime \prime}$ width, fixed values for the heliocentric systemic velocity at $2461 \mathrm{~km} \mathrm{~s}^{-1}$ (RC3), inclination angle at $i=50^{\circ}$ (a mean between the values of Downes et al. 1996 and Regan et al. 1996) and position angle at $188^{\circ}$ (the value found by Regan et al. 1996) and obtained $\left(x_{0}, y_{0}\right)$ using Eq. (1). We found a large scatter in the values of the kinematic centre for annuli far from the centre of the galaxy. This is due to the paucity of $\mathrm{H} \alpha$ emission in the disc of the galaxy outside the arms and to the fact that lower resolution data cubes are more spatially affected by the smoothed non-circular motions along the bar in the data cubes. The mean value of $\left(x_{0}, y_{0}\right)$ for the rings inside $12^{\prime \prime}$ corresponds to the coordinates $4^{\mathrm{h}} 23^{\mathrm{m}} 26.94^{\mathrm{s}}\left( \pm 0.02^{\mathrm{s}}\right) 75^{\circ} 17^{\prime} 44.0^{\prime \prime}\left( \pm 0.1^{\prime \prime}\right), \mathrm{J} 2000$. We checked this value using the same procedure for the velocity maps of lower and higher $\left(10^{\prime \prime}\right.$ and $\left.2^{\prime \prime}\right)$ resolution, and found that the differences in the rotation centre from the value adopted were inside the error bars.

The systemic velocity was obtained from the $10^{\prime \prime}$ resolution velocity map with rings of width $5^{\prime \prime}$. The centre position was fixed to the value found previously, and the inclination and position angle were fixed to the same values used to derive the rotation centre. The mean value for $v_{\text {sys }}$ in all the rings

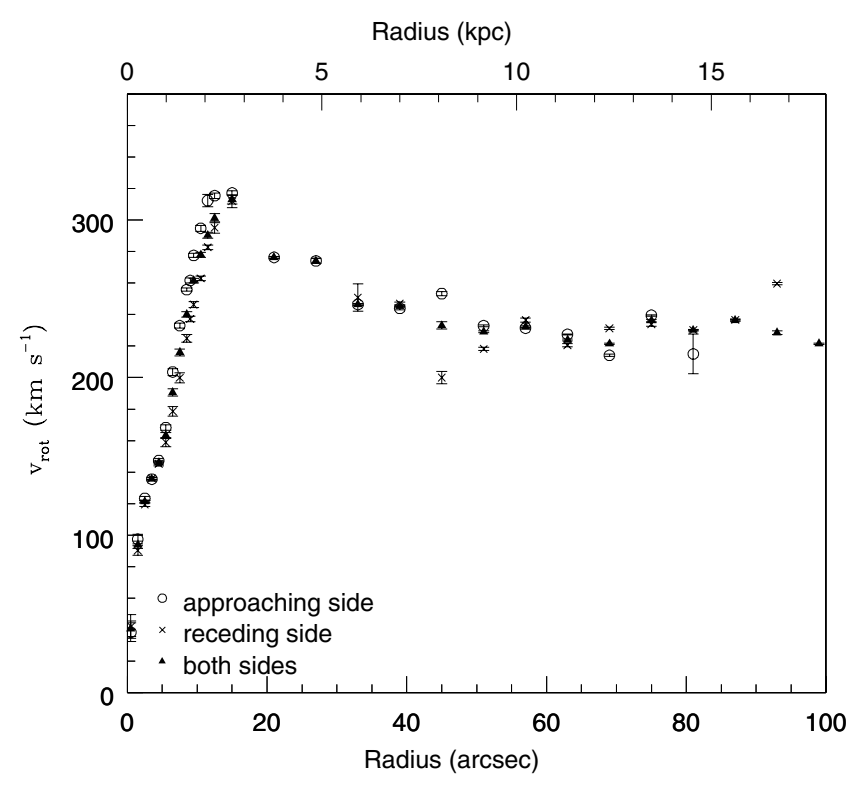

Fig. 2. H $\alpha$ rotation curve of the disc of NGC 1530, for the receding and the approaching sides of the galaxy separately. The agreement between the two sides shows the symmetry of the velocity field and the reliability of the rotation centre.

is $2466 \pm 4 \mathrm{~km} \mathrm{~s}^{-1}$, where the error is the standard deviation from the mean. This value is in agreement with the value found by Reynaud et al. (1997) of $v_{\text {sys }}=2466 \mathrm{~km} \mathrm{~s}^{-1}$ for CO data, but differs from that obtained by Regan et al. (1996) in $\mathrm{H} \alpha$, $v_{\text {sys }}=2447 \pm 2 \mathrm{~km} \mathrm{~s}^{-1}$. A way to test whether the rotation centre and the systemic velocity are well determined is shown in Fig. 2, where we have plotted rotation curves for the receding and approaching halves of the galaxy derived separately using the rotation centre and systemic velocity found above. The coincidence of the velocity points for both sides, especially in the central zone of the galaxy, provides evidence of the symmetry of the velocity field and the validity of the values for the rotation centre and systemic velocity. We also derived the rotation curves for the receding and approaching halves with the rotation centre found in Regan et al. (1996), but in that case we found discrepancies which appeared unphysically large. It is the better resolution of our data ( $1^{\prime \prime}$, while Regan et al. 1996 has a net resolution of $4^{\prime \prime}$ ) which allows us to obtain a more accurate position for the rotation centre.

Finally, we obtained the rotation curve using the velocity map at $2^{\prime \prime}$ resolution in Fig. 1b. For the internal part of the galaxy, out to $12.5^{\prime \prime}$, we used rings of width $1^{\prime \prime}$, and rings of width $6^{\prime \prime}$ for larger radii. The rotation curve obtained in this way is shown in Fig. 3, where the PA and the inclination were fixed at $188^{\circ}$ and $50^{\circ}$ respectively. Figure 3 also displays the rotation curve from Regan et al. (1996), which is similar to that found here, but we obtain higher values of the rotation velocity in the inner part, $10^{\prime \prime}-15^{\prime \prime}$, and also in the $70^{\prime \prime}-90^{\prime \prime}$ radial range. In order to check the PA adopted, we left it as a free parameter in the fit, finding that the values given by the program for most of the annuli are close to $188^{\circ}$ and that the rotation curve did not change significantly from the curve with a fixed PA. The adopted inclination angle $i=50^{\circ}$ was also checked. We fitted $v_{\text {rot }}$ and $i$ with Eq. (1), keeping the other kinematic 


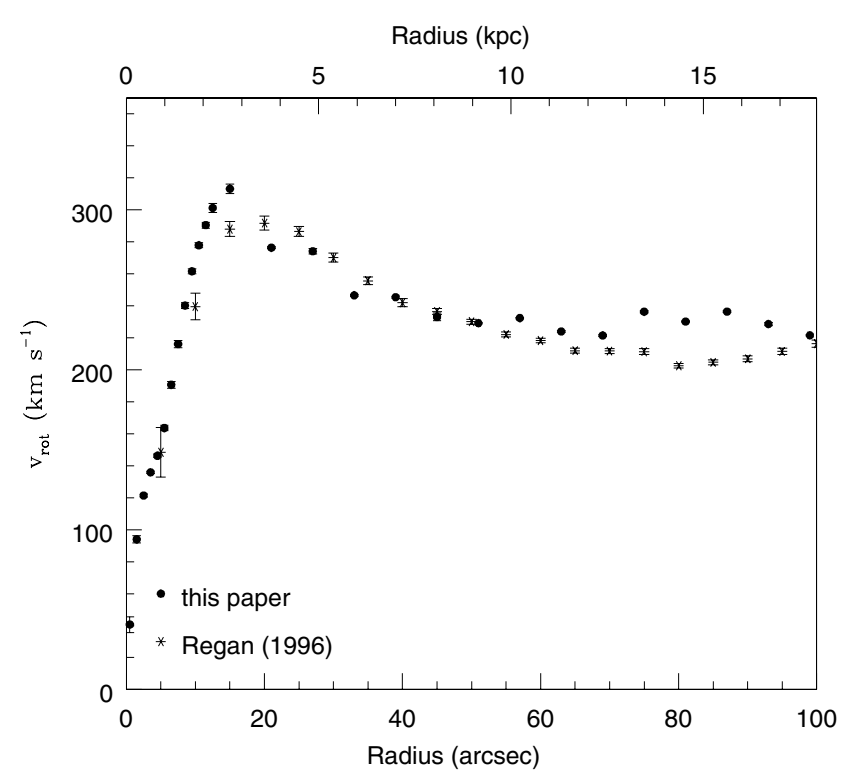

Fig. 3. $\mathrm{H} \alpha$ rotation curve of the disc of NGC 1530, up to $18 \mathrm{kpc}$ from the galactic centre. The rotation curve was obtained by fitting tilted rings to the observed velocity field in Fig. 1b. Also shown is the rotation curve obtained by Regan et al. (1996).

parameters fixed, and found a mean value of $i=(53 \pm 8)^{\circ}$, in good agreement with the value adopted.

To test the importance of a careful definition of the freeangle we reduced its value to $20^{\circ}$ and re-derived the rotation curve. As seen in Fig. 4 this produced "rotational velocities" rising to values of over $500 \mathrm{~km} \mathrm{~s}^{-1}$, as there is an obvious contribution from the non-rotational motion along the bar projected into the line of sight. As we will see below the amplitude of the gas motion along the bar is over $100 \mathrm{~km} \mathrm{~s}^{-1}$, and it is easy to see that this will yield the unrealistically high velocities shown in Fig. 4. Fortunately, the bar orientation in NGC 1530 does not make it too difficult to isolate and avoid the emission from gas which contains a significant non-circular velocity component, but Fig. 4 offers a warning to those using maps of much lower resolution in similar circumstances.

\subsection{The residual velocity map}

After deriving the mean rotation curve, an easy way to explore non-circular motions within the galactic disc is to make a twodimensional projection of this curve and subtract it from the observed velocity field. The two-dimensional projection of the initial rotation curve is seen in Fig. 1c, where we have superposed an isophote map of intensities to show the positions of the emitting components. The projection has classical symmetry, but shows a few small features: "wiggles" which occur at some three quarters of the radial distance from the centre to the edge of the field. These can be explained as effects of streaming motions in the spiral arms, which have not been subtracted adequately from the mean rotation curve.

While it is difficult to get an exact separation of rotation and streaming motions, we have made a first approximation by assuming that the true rotation curve is smooth. Applying a second order polynomial fit to the part that is observed as smooth,

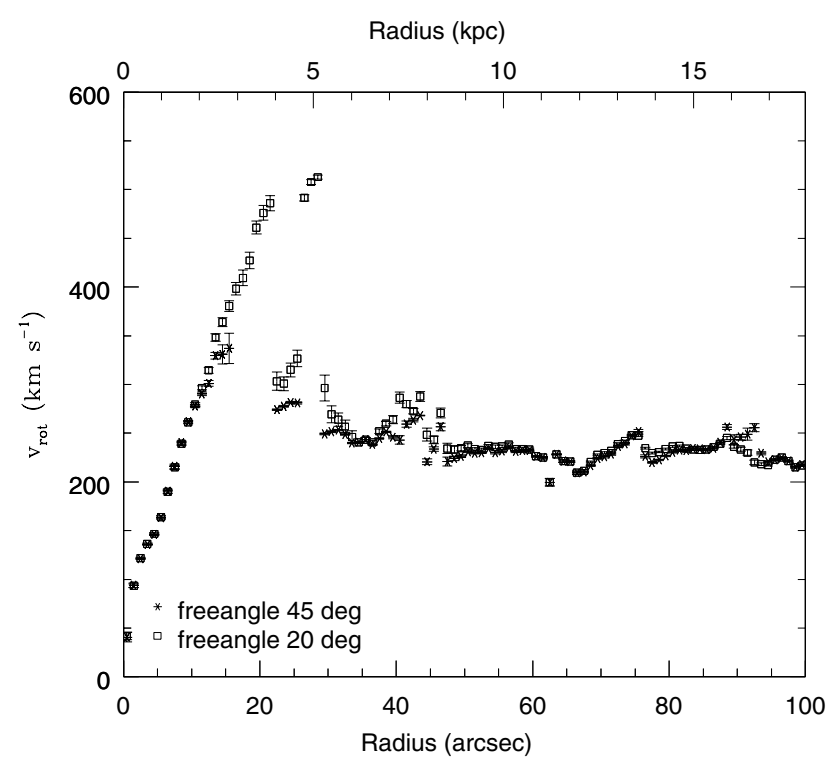

Fig. 4. $\mathrm{H} \alpha$ rotation curves of the disc of NGC 1530 obtained with different "free-angles" and with rings of 1 " width. For free-angles less than $\sim 30^{\circ}$ there is an obvious contribution from the non-rotational motion along the bar in the mean calculated rotational velocity that can "contaminate" the rotation curve of the galaxy.

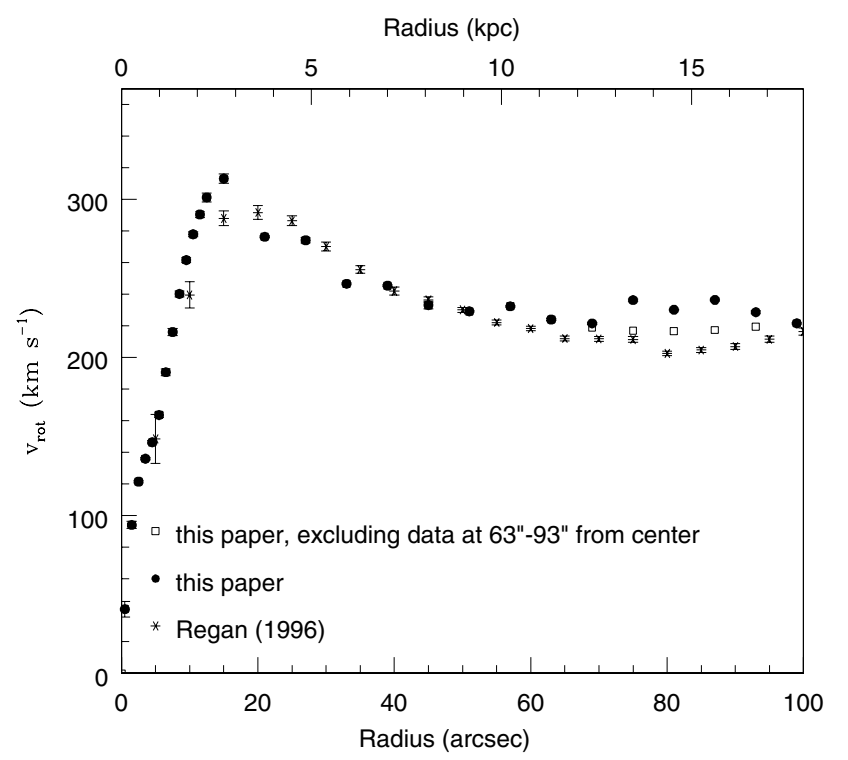

Fig. 5. H $\alpha$ rotation curve of the disc of NGC 1530. This rotation curve has been obtained by subtracting the effect of the streaming motions in the spiral arms of the galaxy from the mean rotation curve shown in Fig. 3 (see text for further details).

i.e. between $27^{\prime \prime}$ and $63^{\prime \prime}$ from the centre, we then extrapolate this to cover the range between $63^{\prime \prime}$ and the edge of the field at $99^{\prime \prime}$ from the centre, and go on to combine it with the original curve out to 63 " radius, to give the final "true" rotation curve. The result of this procedure is shown in Fig. 5, where we have compared our original rotation curve with the new curve and also with the curve by Regan et al. (1996). Our procedure has isolated the wiggles between 63" and 93".

We can then proceed to produce a new two-dimensional projected model for the rotation in which the wiggles are no 
longer present, and to subtract off this new rotation model from the original velocity field, yielding a resulting map of residuals as shown in Fig. 1d. There are clearly ordered fields of noncircular velocity both around the bar, and in the arms, but we can check on the model subtraction by examining the residuals of the regions which are in the disc outside the arms and bar. These in general show, as they should if the rotation curve subtraction is valid, net residual velocities close to zero: less than $\sim 10 \mathrm{~km} \mathrm{~s}^{-1}$, which is close to the precision limit of the observations. We can now see clearly the streaming motions across the arms in Fig. 1d. These have projected values between 10 and $30 \mathrm{~km} \mathrm{~s}^{-1}$, with similar amplitudes in both arms.

\subsubsection{The residual velocities along the bar}

Of most striking interest is the fairly complex non-circular velocity field associated with the bar. Approaching velocities of up to $120 \mathrm{~km} \mathrm{~s}^{-1}$ are observed along the north side of the bar, and receding velocities of the same amplitude along the south side. These values, and the implied vectorial direction in elongated quasi-elliptical orbits around the bar with long axis parallel to the bar axis, are in line with classical predictions of the dynamical effects of a stellar bar on the rotating gas which date back to Huntley et al. (1978) and Roberts et al. (1979) as well as to more recent studies by Athanassoula (1992). Most of the observational work has been performed either in H I or more recently in $\mathrm{CO}$ emission. There have been relatively few $\mathrm{H} \alpha$ studies of this phenomenon, for two probable reasons: slit spectroscopy is costly in time if more than a couple of position angles are taken (one good early study in this mode using six slit positions on NGC 1512 was published by Lindblad \& Jorsater 1981); and $\mathrm{H} \alpha$ emission is much patchier than either $\mathrm{HI}$ or CO. However, as we can see from the data in the present article, the use of a scanning Fabry-Pérot for this work permits full two-dimensional coverage at an angular resolution better than that of essentially all $\mathrm{H} \mathrm{I}$ or $\mathrm{CO}$ measurements, which can compensate to an interesting degree for the intrinsic patchiness of the observed field.

In Fig. 1d there are several complexities in the non-circular velocity field which are worth pointing out. Taking the north side of the bar we can see a zone with high bar-streaming velocities, of order $120 \mathrm{~km} \mathrm{~s}^{-1}$ in a direction approaching us to the east of the centre, and another region, more restricted in size and velocity range, with similar velocities to the west. To the south of the bar we can see a diametrically symmetric flow, with high receding velocities, again of order $120 \mathrm{~km} \mathrm{~s}^{-1}$ receding on west side, and two smaller zones with similar velocities to the east. It is tempting to consider these flows as direct evidence for motion aligned with the bar and terminating in zones of shock towards the ends of the bar. We will see later that there is considerable evidence in favour of this, and of the direct causal links between properties of these flows, and both the stimulation and inhibition of massive star formation.

Examining Fig. 1d, the question arises as to how the flow can apparently be stopped half way along the bar, yet continue on beyond the central zones as described above. This appearance does not give a true picture of the velocity field and is essentially due to the patchiness of the emission. However taking the latter into account we can interpret the observations in terms of a resonance model of the type illustrated in Downes et al. (1996) to interpret their measurements in CO, based on models incorporating an ILR to a main galactic bar by Athanassoula (1992). Qualitatively, these models show how the bar dynamics produces orbits termed $x_{1}$, whose major axes are aligned with the major axis of the bar, but that the presence of an inner Lindblad resonance within the bar structure (not far from the nucleus) implies that gas will move on the perpendicular $x_{2}$ elliptical orbits within the ILR. The behaviour of a particular gas cloud in an $x_{1}$ orbit will then depend critically on its impact parameter with respect to the nucleus, i.e. on the length of its orbital semi-minor axis. Gas on orbits close to the bar major axis will be dragged into $x_{2}$ orbits as it passes close to the nucleus, and will not escape along the bar. However, gas on orbits well away from the major axis will remain essentially on those orbits until it reaches the far end of the bar, where it will be braked and shocked. Gas on intermediate orbits will swing in towards the bar major axis, where in turn it will also be braked and shocked. In Fig. 8 we have illustrated these types of orbital behaviour, with a representative set of five trajectories on either side of the bar. Gas moving on the innermost trajectory will be pulled into an $x_{2}$ orbit, while gas on the others will end up impinging on the bar progressively further along, as their initial impact parameters increase. Because the velocity vector is observed only where star formation is occurring, we cannot follow these trajectories along their lengths, but see clearly their initial and final points in the residual velocity field.

\subsubsection{Residual velocity cross-sections along and perpendicular to the bar}

In Figs. 6a-d we show profiles in residual velocity perpendicular and parallel to the bar direction $\left(\mathrm{PA}=116^{\circ}\right)$. The positions and directions of the cross-sections chosen are plotted over the residual velocity map in Fig. 7. For profiles perpendicular to the bar and located where the bar connects with the spiral arms $\left(-40^{\prime \prime}\right.$ in Fig. 6a and $40^{\prime \prime}$ in Fig. 6b) the absolute value of the residual velocity along the line of sight is $\sim 70-80 \mathrm{~km} \mathrm{~s}^{-1}$. The velocities rise as we approach the centre of the bar and at $\pm 30^{\prime \prime}$ are $\sim 100 \mathrm{~km} \mathrm{~s}^{-1}$. We note particularly the symmetry of the profiles on either side, at $\pm 20^{\prime \prime}$ of the profile through the nucleus. We can also see very clearly the steep velocity gradients at these positions. In the profiles parallel to the bar (Figs. 6c and d) there is also evidence of rapid velocity changes. In particular the profiles at $4^{\prime \prime}$ and $8^{\prime \prime}$ in Fig. $6 \mathrm{c}$, and $-12^{\prime \prime}$ and $-8^{\prime \prime}$ in Fig. 6d, show very sharp velocity gradients. These gradients mark a combination of braking and projection effects: braking effects as the gas flow along the bar meets the central gas concentration, and projection effects as the flow vector switches direction from nearly along the line of sight (along the bar) to nearly perpendicular to the line of sight (as the gas is pulled into circumnuclear orbits). A result of examining these profiles was to induce us to make a more detailed map of the velocity gradients, which we will show and discuss below. 

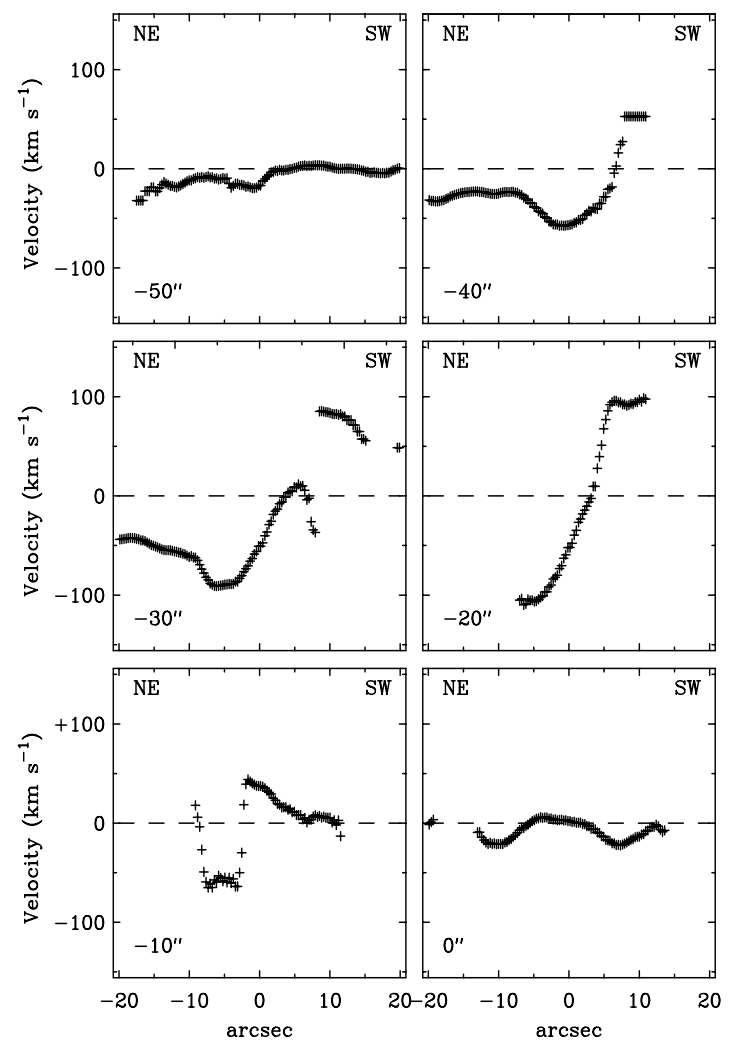

(a)
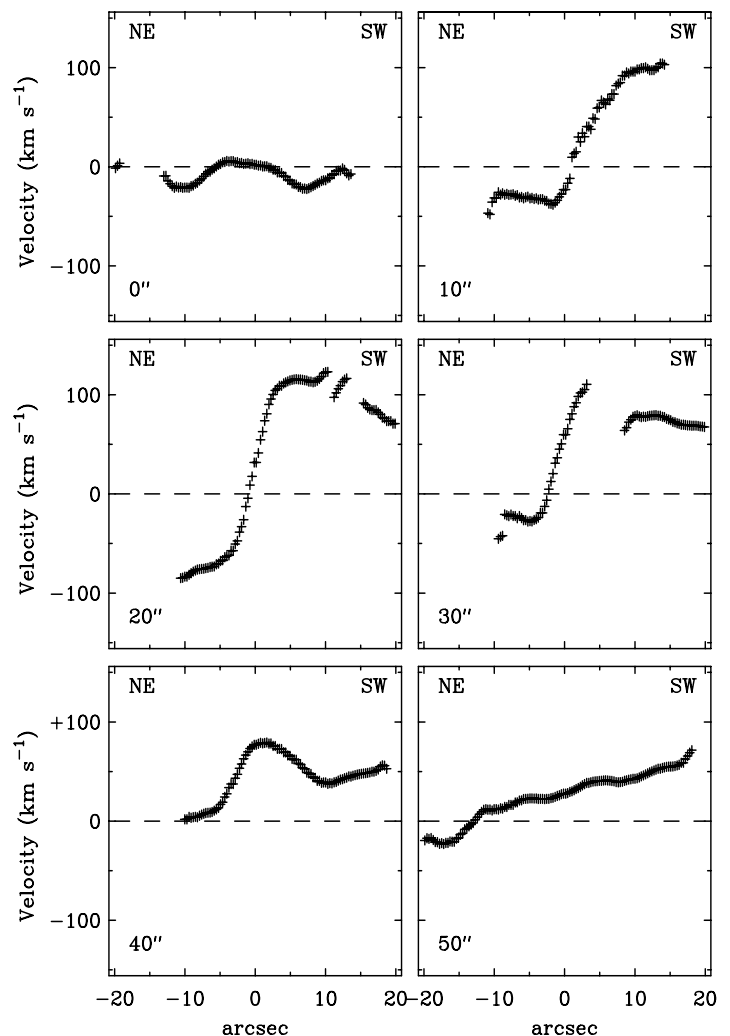

(b)
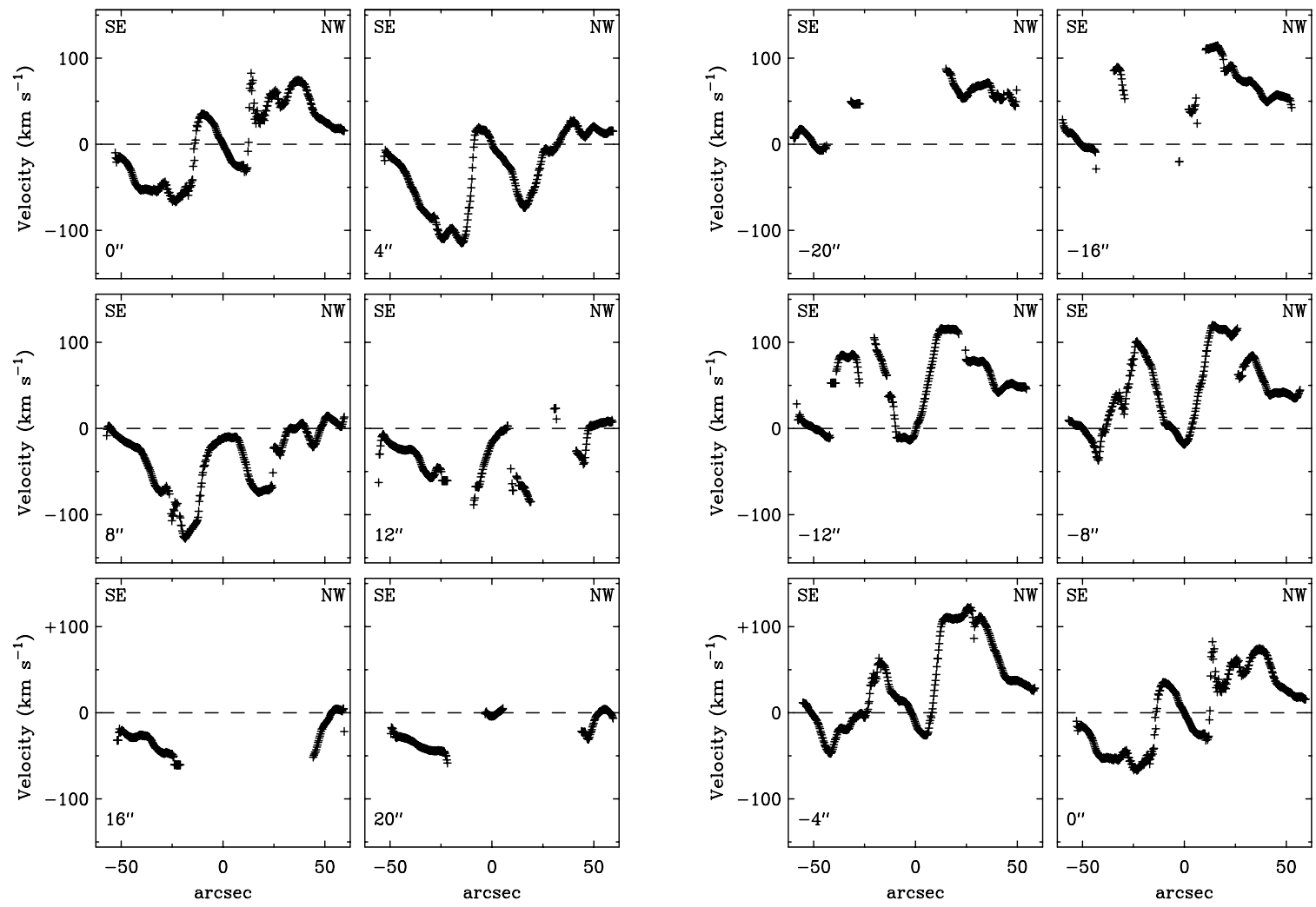

(c)
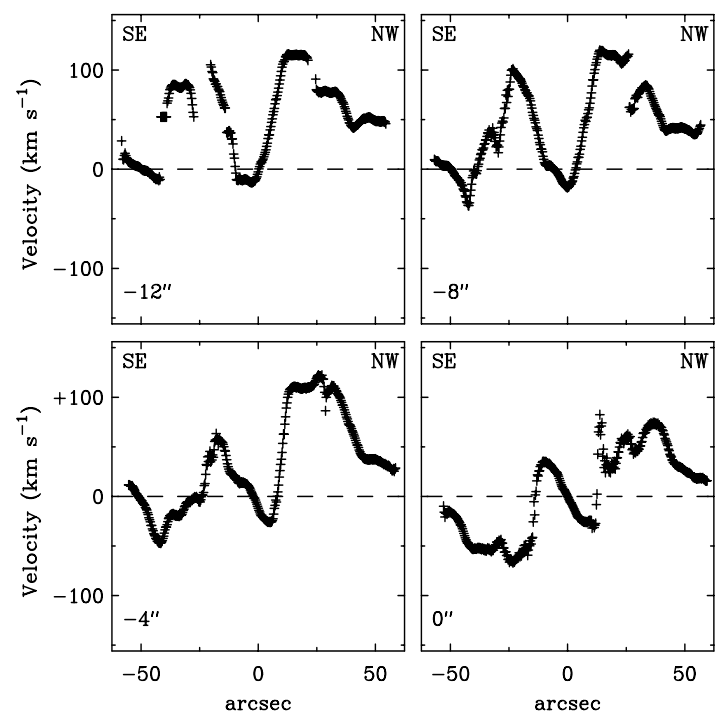

(d)

Fig. 6. Profiles of the residual velocity perpendicular to the bar of the galaxy: to the SE a) and to the NW b), and parallel to the bar: to the NE c) and SW d). The profile tracks are located towards the left, right, top and bottom of Fig. 7 in panels a), b), c) and d), respectively. The distances of the profile tracks to the galaxy kinematic centre are indicated in the bottom-left hand corner of each plot. 


\subsection{Residual velocity gradient maps}

Following up the indication of the presence of steep velocity gradients in Figs. 6a-d, we opted to produce a full map of the velocity gradients in the system, taking advantage of our excellent angular resolution. To make sure that the steep gradients were not artefacts of our data processing we first used the $2^{\prime \prime}$ resolution data cube, and made position-velocity diagrams at those points where Figs. 6a-d indicated that we ought to find rapid velocity changes. This exercise was non-trivial, since the steepest gradients occur in zones of low $\mathrm{H} \alpha$ intensity, for reasons which we will discuss further below. The diagrams are shown in Fig. 10, where we can see at once that the velocity gradients are real and very steep, at the positions chosen. We go on to derive maps in velocity gradient, selecting two directions, parallel and perpendicular to the bar, to optimize the information about the gas flows induced by the gravitational perturbation of the bar. The gradient for a given direction was derived in a straightforward way as follows: the velocity residual map was displaced by one pixel in that direction, and subtracted off from the original map; this procedure was repeated in the opposite direction, and the gradient was found by co-adding the absolute values for the two maps, and normalizing the result, dividing by two. The same procedure was followed for both of the selected directions, yielding two independent velocity gradient maps. The whole map of residual velocity gradient in the direction perpendicular to the bar is shown in Fig. 9a, while in Figs. 11d and c we show maps of the gradients in residual velocity, measured in the directions perpendicular to the bar and along the bar respectively, in the zone of the bar. The regions of steep gradient show up very clearly, and reach high values $\left(\sim 0.35 \mathrm{~km} \mathrm{~s}^{-1} \mathrm{pc}^{-1}\right)$. Near the centre of the bar we can pick out elongated regions where the motions along the bar axis are in counterflow, so that an amplitude of over $100 \mathrm{~km} \mathrm{~s}^{-1}$ in one direction rapidly gives way to the same amplitude in the opposite direction. The measured gradients imply that this occurs over distances of a few hundreds of parsecs, close to the limit of direct detection, so that there may in fact be much steeper shocks than this. Our decomposition of the gradients into these two perpendicular directions does not effectuate a very clean separation between different intrinsic dynamical effects, above all because the observations give the projected components along our line of sight, and projection effects are not removed. This would be difficult to do correctly without a previous underlying model, which would assume too much about the directions of motion to be reliable, so we prefer to leave the observations as they are. In general, zones of high velocity gradient in one direction also show high gradients in the perpendicular direction, though the details differ on very small scales.

In Fig. 9b we have also included an image of NGC 1530 in the $V$ band. The morphological and positional agreement between the dust lanes and the zones of maximum velocity gradient perpendicular to the bar illustrated in this figure is striking. This result may not be qualitatively unexpected, since various models of gas flows and shocks in barred galaxies do predict that gas and dust densities should be elevated in the shocked zones. However, to our knowledge, this is the first presentation of a direct one-to-one mapping of the velocity gradients in shocks and the dust lanes along a bar with comparable angular resolution.

Comparing these velocity gradient maps, and in particular the map of gradients perpendicular to the bar, Figs. 9a or 11d, with the total intensity map in Fig. 1a we can see clearly that the star formation is peaking in zones where the velocity gradients are low. There is a very good anticorrelation here, which holds over the whole of the face of the galaxy: in the arms, along the bar, and in the central zone. In general, when comparing the non-circular velocities in Fig. 11e with the velocity gradients in Figs. 11c, d, we find high velocity gradients associated spatially with significant non-circular velocities, but there are two specific zones where this is clearly not the case. Along the NW side of the bar there are two bright star-forming regions, the less intense of which is situated within gas with a projected residual flow vector of amplitude $\sim 100 \mathrm{~km} \mathrm{~s}^{-1}$, as seen in Fig. 11e. This same zone, is clearly in a velocity gradient minimum as seen in Figs. 11d and e. A second example is the intense star forming zone where the bar meets the NW arm, where the velocity gradients are low, but the net velocity vector has an amplitude of $\sim 30 \mathrm{~km} \mathrm{~s}^{-1}$. Both of these are regions with strong star formation, and low velocity gradient but relatively high residual velocity. We will discuss the implications of these correlations and anticorrelations in Sect. 4.

\subsection{The velocity dispersion}

The second moment of the intensity-wavelength distribution represents the velocity dispersion of the emission line observed. Since we are interested in the dispersion due to the turbulent (non-thermal) motions of gas, we must extract it from the observed $\mathrm{H} \alpha$ emission line profiles taking into account effects which also contribute to broaden the observed line shape. These are the natural $\mathrm{H} \alpha$ emission line broadening, thermal broadening and instrumental broadening. We subtracted these components in quadrature from each point of the observed velocity dispersion map, to obtain a new map of the dispersion due to the non-thermal motions of the ionized gas. For further details on the procedure see Rozas et al. (1998, 2000). In Fig. 11f we show the map of non-thermal velocity dispersion in the zone of the bar. In general there is a close correlation between high surface brightness (as seen in Fig. 11a) and high velocity dispersion, as expected knowing that much of the measured turbulence is stimulated by the energy output of young massive stars, which also give rise to the $\mathrm{H} \alpha$. There are some zones, notably towards the ends of the bar, where this agreement is less clear, i.e. where the ratio of the $\mathrm{H} \alpha$ surface brightness to the measured dispersion is much lower than for other star forming zones. We attribute this to the fact that our measurements of dispersion cannot avoid including components of steep localized velocity gradients which are particularly important where the arms break away from the bar. As we will see in Sect. 4.2, there is a clear anticorrelation between the local star formation rate and the gas velocity gradient, and the observations of dispersion alone would not easily pick this up. 


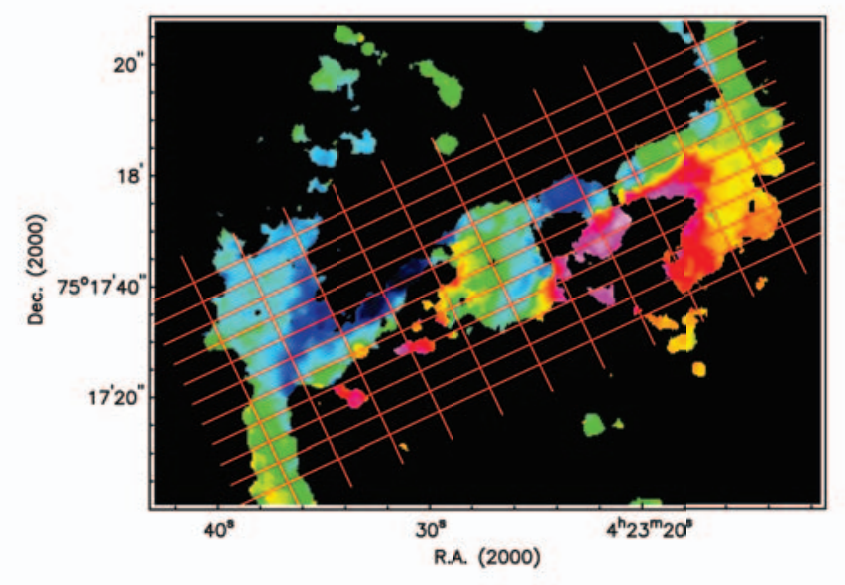

Fig. 7. Lines tracing the cross-sections perpendicular and parallel to the bar of NGC 1530 represented in Figs. 6a to $6 \mathrm{~d}$ over a portion of the residual velocity map.

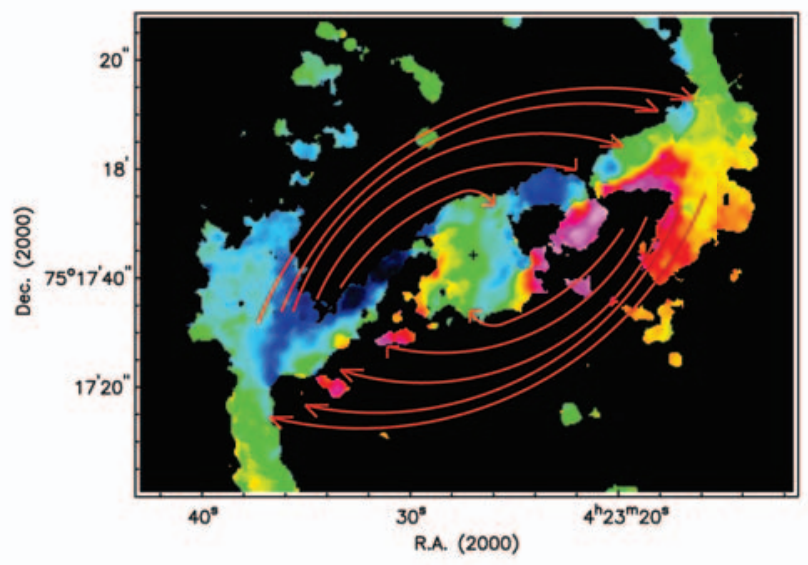

Fig. 8. Schematic representation of the gas orbits along the bar on the residual velocity map, illustrating the dependence of the gas trajectories on orbit semi-minor axis.

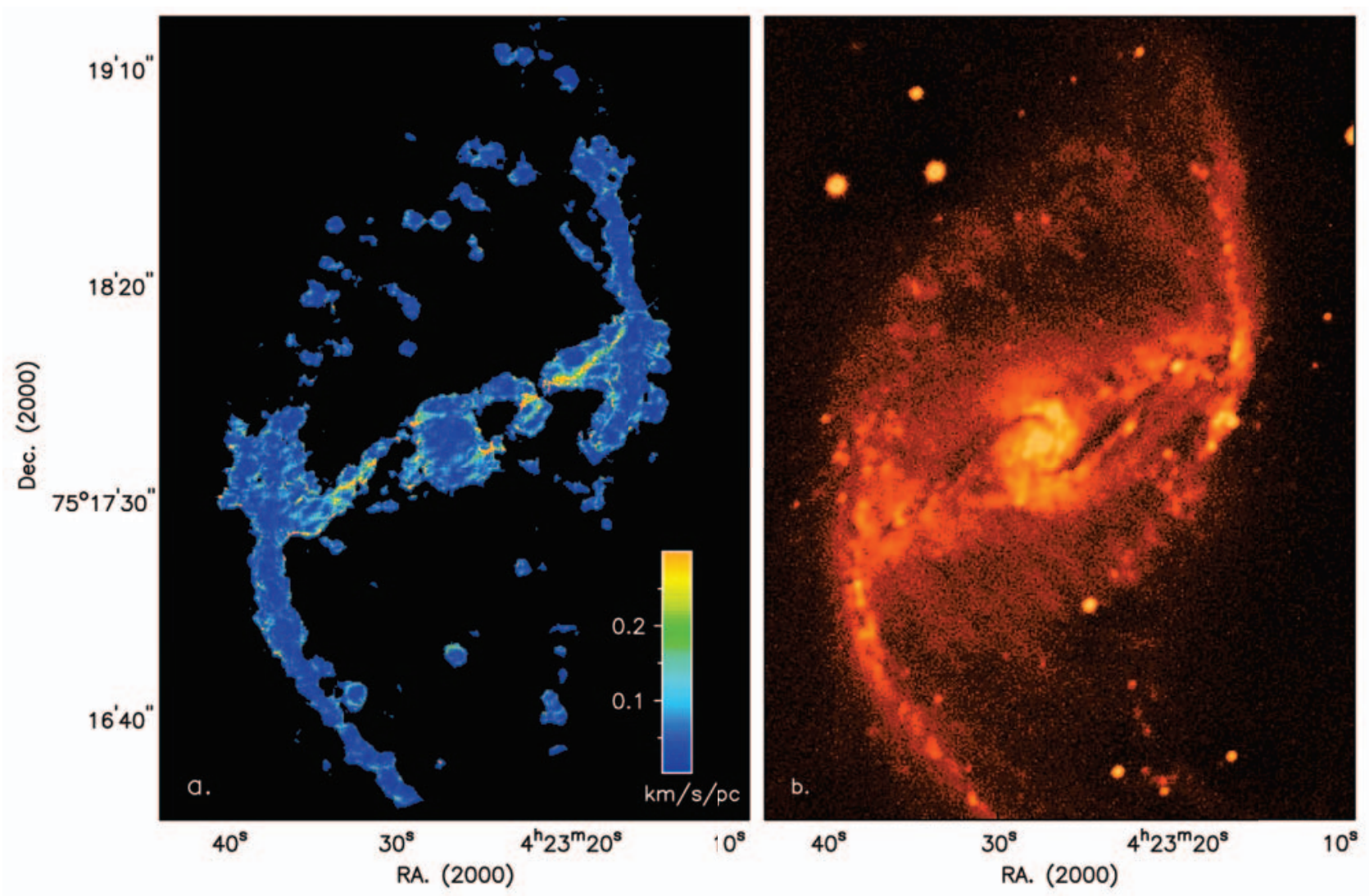

Fig. 9. a) Velocity gradient map perpendicular to the bar. Zones of maximum shear are clearly seen (cf. the dust lanes in Fig. 9b). b) Image of NGC 1530 in the optical $V$ band obtained with the KPNO 4-m Mayall telescope (NOAO/AURA/NSF).

\section{Relationships between the velocity field and the local star formation rate}

\subsection{Bar cross-sections in velocity and $\mathrm{H} \alpha$ surface brightness}

To examine more closely the relation between the current star formation rate as measured in $\mathrm{H} \alpha$ surface brightness, and the velocity field of the gas in the region of the bar, we obtained a series of cross-sections in which the residual velocity can be compared directly with the $\mathrm{H} \alpha$ surface brightness, as displayed in Figs. 12a, and $\mathrm{b}$ with the velocity dispersion. In Fig. 12a we show a set of these cross-sections perpendicular to and parallel to the bar, comparing surface brightness with residual velocities, i.e. non-circular velocity. As a general feature of 


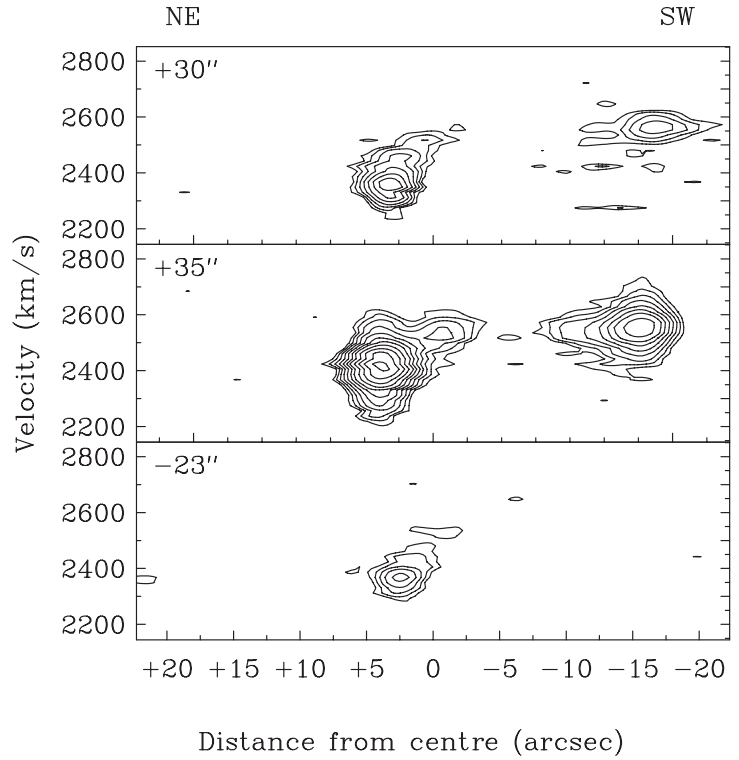

Fig. 10. Position-velocity diagrams taken along cross-sections perpendicular to the centre line of the bar. Distances from the nucleus are shown in the top left hand corner. Distances are positive towards the NW and negative towards the SE.

the cross-sections it is clear that there is an anti-correlation between $\mathrm{H} \alpha$ intensity and residual velocity ${ }^{1}$. We can make a plausible hypothesis that suggests two distinct phenomena, one of which inhibits star formation and the other of which helps to produce it, which combine to yield this general result. Around the bar, the long elliptical orbits should have (see e.g. Huntley et al. 1978) velocity vectors around the ellipse which are high close to the bar major axis, and fall further and further from the axis. In this configuration the maximum residual velocity around the bar should be close to the zone of maximum velocity shear. The conditions which determine the stability of clouds for global star formation in galaxy discs have been shown by Kennicutt (1989), following Toomre (1964), to depend on the relationship between the gas surface density and the velocity dispersion in the plane. However, this general condition should be affected by the presence of velocity shear, which would act against the condensation of massive gas clouds to form stars, as suggested by Reynaud \& Downes (1998). Therefore, in the global motion around the bar we might expect to find a lower star formation rate in zones of high velocity. However, within this global framework, certain shocked zones are predicted to be places where star formation is accelerated (see e.g. Elmegreen et al. 2002), and where the global motion is locally strongly braked. This will give rise to zones of low velocity and high star formation rate which also contribute to a negative correlation between gas velocity and star formation rate, but with variations over more compressed spatial scales. The global anti-correlation is well seen in all the cross-sections of Fig. 12a, where peaks in the $\mathrm{H} \alpha$ surface brightness nearly all

1 To make for a homogeneous comparison we have plotted as positive the amplitude of the velocity vector without reference to its direction, while from the map in Fig. 1d it should be clear that the velocities are in opposite directions along opposite sides of the bar. coincide with troughs in the residual velocity. Local effects are especially well seen in the cross-section through the nucleus parallel to the bar, which cuts two shock fronts; near these the star formation rate is low, but in the radial galactocentric range between them it is very high. These relationships are also seen in Fig. 12b, where we have made cross-sections along the same tracks, perpendicular to and parallel to the bar, but where we have plotted the velocity dispersion instead of the $\mathrm{H} \alpha$ surface brightness. Qualitatively the graphs are very similar, with a similar set of anticorrelation patterns. The main difference is that the velocity dispersion peaks tend to be broader than those in surface brightness, reflecting the turbulent velocity structure within individual ultraluminous $\mathrm{H}$ II regions (there is a clear tendency for the brighter regions to have the biggest turbulent widths; see e.g. Rozas et al. 1998; Relaño et al. 2003b, in preparation). We should also note velocity gradients on different scales, in particular gradients in the global velocity pattern can contribute to an observed local value of the velocity dispersion, while there is no analogous effect for surface brightness. There is quantitative material in these data which can contribute to theoretical modelling of scenarios for massive star formation, though this is beyond the direct scope of the present article.

\subsection{Bar cross-sections in velocity gradient and $\mathrm{H} \alpha$ surface brightness}

We saw qualitatively, by inspection in Sect. 3.3, that where the velocity gradients in the bar are high, star formation appears less intense. For a more quantitative appreciation of this effect we have made graphs of residual velocity gradient and $\mathrm{H} \alpha$ intensity, taken along scans perpendicular to and parallel to the bar. We have used both Figs. 11c and d for this, so that we are comparing $\mathrm{H} \alpha$ surface brightness with velocity gradient parallel to the bar and perpendicular to the bar, respectively in the two cross-sections in Figs. 13a and b.

In both graphs and in all the cross-sections, we note the coincidence between the intensity peaks and local minima, or points of inflection, in the velocity gradient. There is a striking anticorrelation between velocity gradient and star formation rate as represented by the $\mathrm{H} \alpha$ surface brightness, and it is particularly striking in zones of high velocity gradient. In the two zones where the velocity gradient is both high and rapidly varying, between $10^{\prime \prime}$ and $15^{\prime \prime}$ from the centre of the scans parallel to the bar through the nucleus (centre-right plot of Figs. 13a and 13b), there is a notable absence of star formation. These are the shocked zones at the edges of the central $x_{2}$ dominated region, where the flow along the bar is being braked and the gas is changing its orbital configuration. The star formation within the central region is occurring well within this radial range, i.e. well offset from these shocks. As well as the global effects just described, there are local dips in the velocity gradient, of smaller amplitude, coincident with the most intense star forming regions, which reflect the kinematic impact of the gas motions within the individual regions on the overall velocity field. 

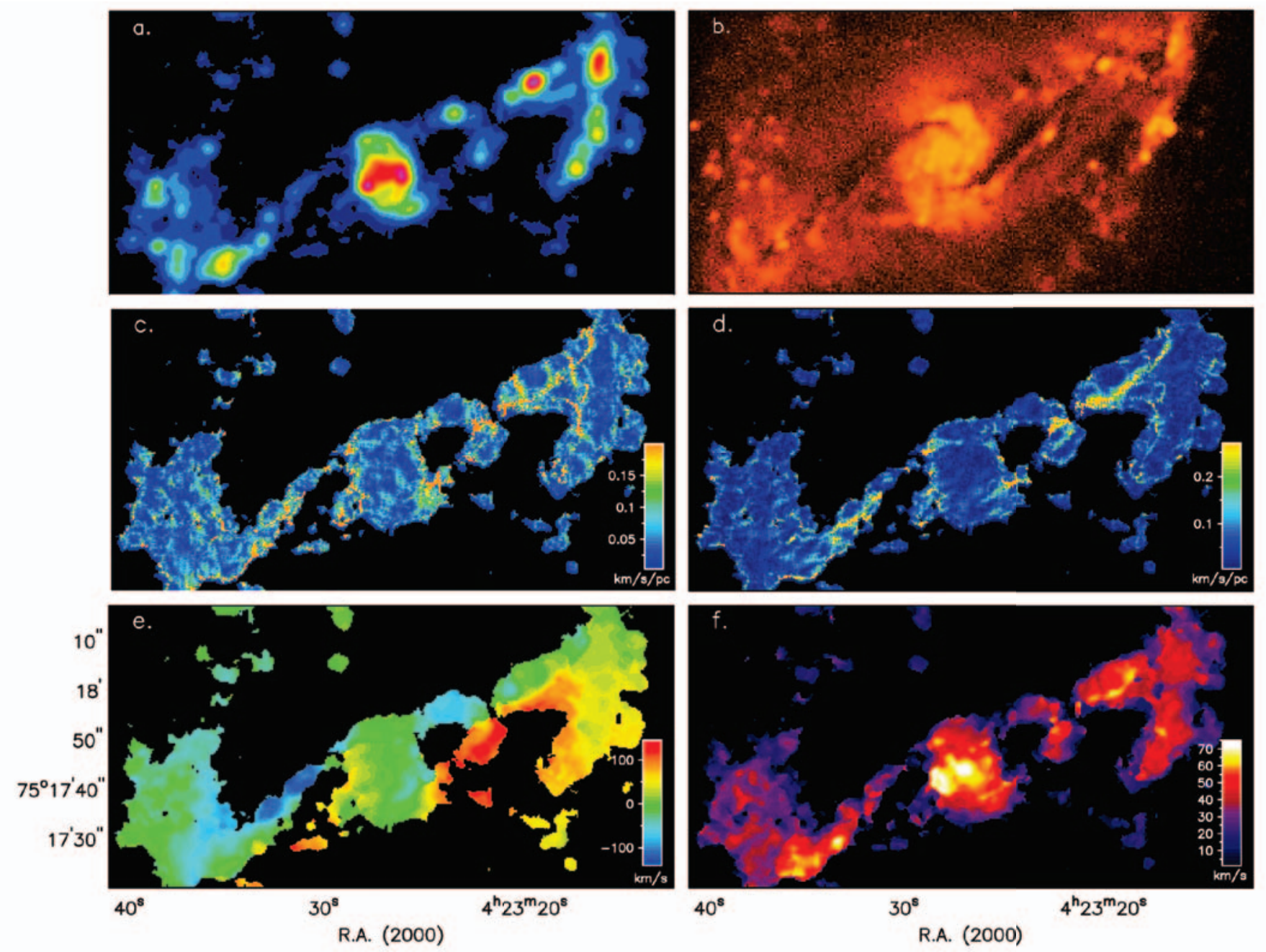

Fig. 11. a) Intensity map of the $\mathrm{H} \alpha$ emission in the zone of the bar. b) $V$ band image. c) Velocity gradients parallel to the bar. d) Velocity gradients perpendicular to the bar. e) Non-circular residual velocity map. f) Non-thermal velocity dispersion map. All images (a) to f)) show the same zone of the galaxy.

\section{Velocity distribution and morphology within the inner disk}

\subsection{The nuclear bulge and the circumnuclear velocity pattern}

Both morphologically and kinematically the central zone, which shows up as a projected disk of major axis radius $\sim 10^{\prime \prime}$, i.e. $\sim 1.8 \mathrm{kpc}$, is easily distinguished in $\mathrm{H} \alpha$ from the bar. At its centre, as well seen in the Hubble Space Telescope (HST)NICMOS image presented in Fig. 14a, there appears to be a very small bulge, less than $2^{\prime \prime}$ in diameter, detectable via its circular isophotal shape. The rotation curve for the galaxy as a whole, which we presented in Fig. 5, shows an apparently enhanced rotational velocity gradient within the inner $\sim 5^{\prime \prime}$, but we had produced this curve on the assumption that the only significant velocity component here is in the tangential direction to circular orbits in the plane of the galaxy.

From Fig. 14a we find that there is no major extra mass component on the scale of this steeper gradient, so we explored the possibility that it is in fact indicating the presence of noncircular motions within this inner disk. To do this we assumed, from the form of the rotation curve as a whole, that the velocity gradient between $5^{\prime \prime}$ and $10^{\prime \prime}$ represents the true rotation curve, and can be interpolated between this portion of the curve and the nucleus. We then subtracted off the rotated interpolated curve, to produce a new residual velocity pattern for the central disk, which is shown in Fig. 14c. We can see from this figure that as well as the previously identified non-circular velocities beyond $10^{\prime \prime}$ from the nucleus, we now have two lobes of oppositely directed velocity, nearly symmetric, to the north and south of the nucleus, with peak projected values of $\sim 50 \mathrm{~km} \mathrm{~s}^{-1}$ at a little under $5^{\prime \prime}$ from the centre. This pattern might be attributable to an inner bar or oval distorsion within this inner disk. To check this out we made an unsharp masked image (see Erwin \& Sparke 2002) from the NICMOS near IR image shown in Fig. 14a, by subtracting off from the original a version smoothed with a Gaussian of 5 pixels half-width $\left(\sim 0.38^{\prime \prime}\right.$ FWHM). The result is shown in Fig. 15, in which we can see a bright nucleus, and a small asymmetric spiral, possibly emerging from a tiny bar, whose major axis appears to be at $\mathrm{PA} \sim 120^{\circ}$, but which is not well enough resolved even at this resolution. 

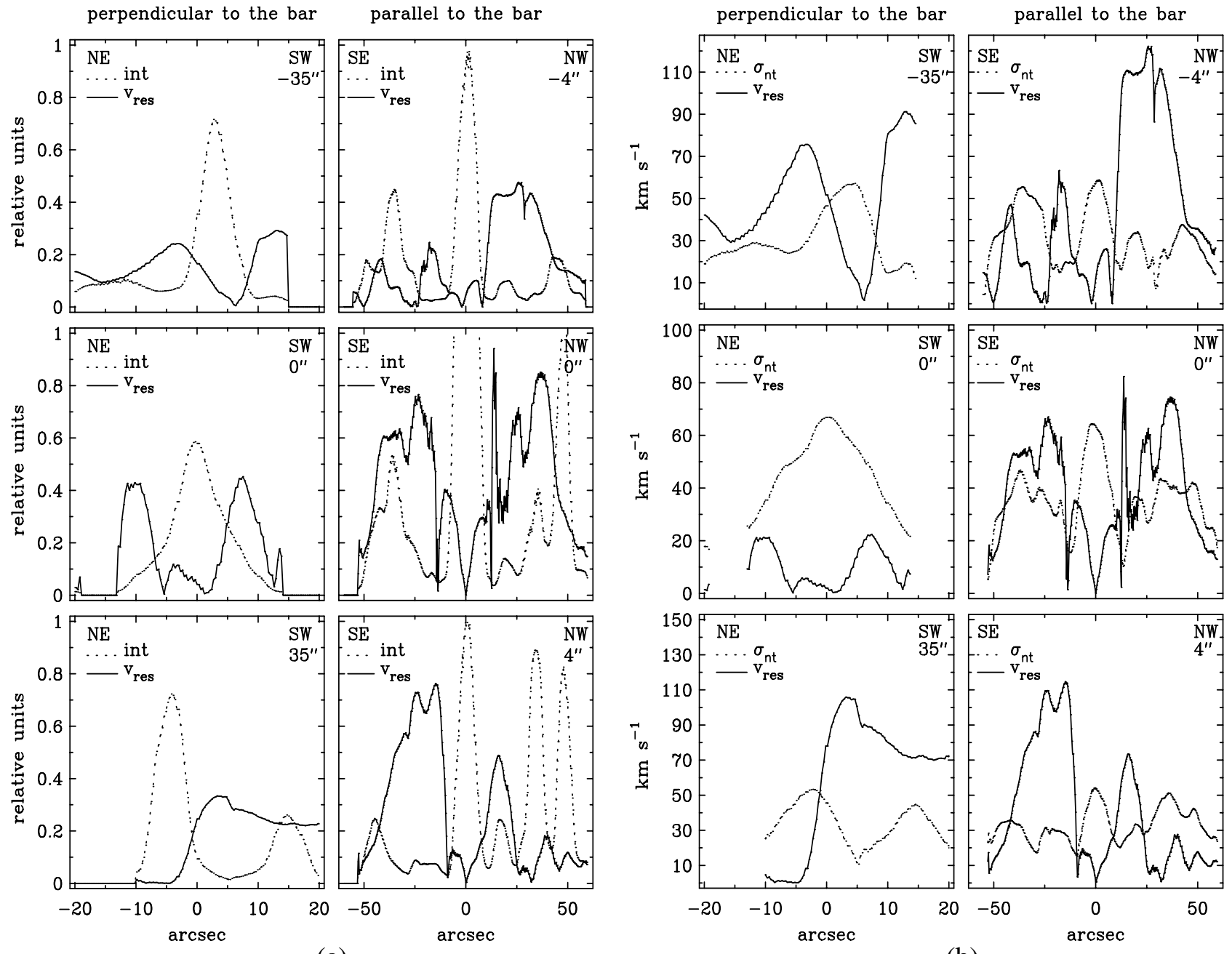

(a)
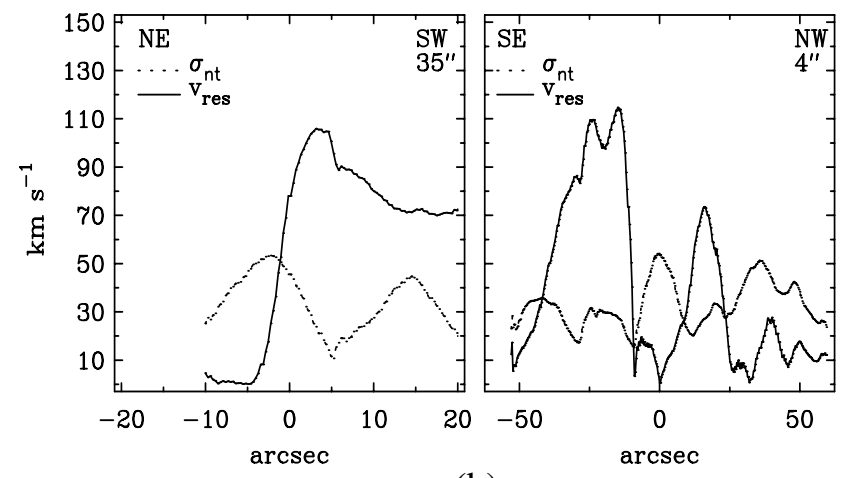

(b)

Fig. 12. a) Profiles of the normalized surface brightness in $\mathrm{H} \alpha$ emission and residual velocity parallel and perpendicular to the bar. The distance of each profile track from the kinematic centre is indicated in the top right corner of each plot. Positive distances in the perpendicular and parallel profiles are respectively towards the NW and NE in Fig. 7. b) Profiles of the absolute residual velocity and non-thermal velocity dispersion parallel and perpendicular to the bar. The tracks of the profiles are the same as those in Fig. 12a.

The dynamical scope of this structure can be appreciated from Fig. $14 \mathrm{c}$, where it has been plotted onto the residual velocity map of the inner disk, and where we can see that it is far too small to be causing the lobed velocity structure, which demands a different explanation.

\subsection{The inner kinematic structure: A spiralling inflow pattern}

We can go on to interpret the inner residual velocity structure as follows. The gas flow along the bar and its deviation into circumnuclear orbits can be followed via the presence of dust lanes which indicate compressed and shocked gas. These dust lanes in the central disk of NGC 1530 were imaged by PérezRamírez et al. (2000; their Fig. 2b) in the near IR, at subarcsec resolution, and are especially well seen in their $J-K$ colour image, which we reproduce here in Fig. 14b. Two clear dust lanes can be seen to the north and south of the inner disk, coming inwards along the direction of the bar major axis, then curling around in orbits which take them to the far side of the nucleus in both cases, where their trajectories have swung through $270^{\circ}$, and terminating on opposite sides of the nucleus at some three arcsec from the centre, along a line with PA $\sim 120^{\circ}$. The positions of the centres of our flow lobes in Fig. 14c coincide with the inward curving dust lanes, so we attribute them to gas flow coinciding with these lanes. The observed velocity structure of the lobes is strongly affected by the fact that we observe the velocities along a single line of sight, and so we detect only their projected components. Where the velocity vector of the inflowing gas is perpendicular to the line of sight the flow cannot be detected. Thus, the lobe to the north of the nucleus is in fact reflecting the final stages of the inflow from the southern edge of the bar, and vice versa. The two flows spiral around one another in an interlocking pattern. As a test of this prediction we have produced a simple kinematic model showing how these flows would appear in projected velocity. We have assumed for simplicity that the intrinsic velocity vector has a constant amplitude, equal to $60 \mathrm{~km} \mathrm{~s}^{-1}$, and projected it into the line of sight to the observer, using the inclination and position 


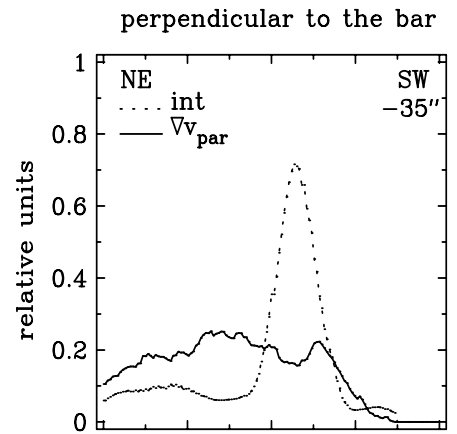

parallel to the bar
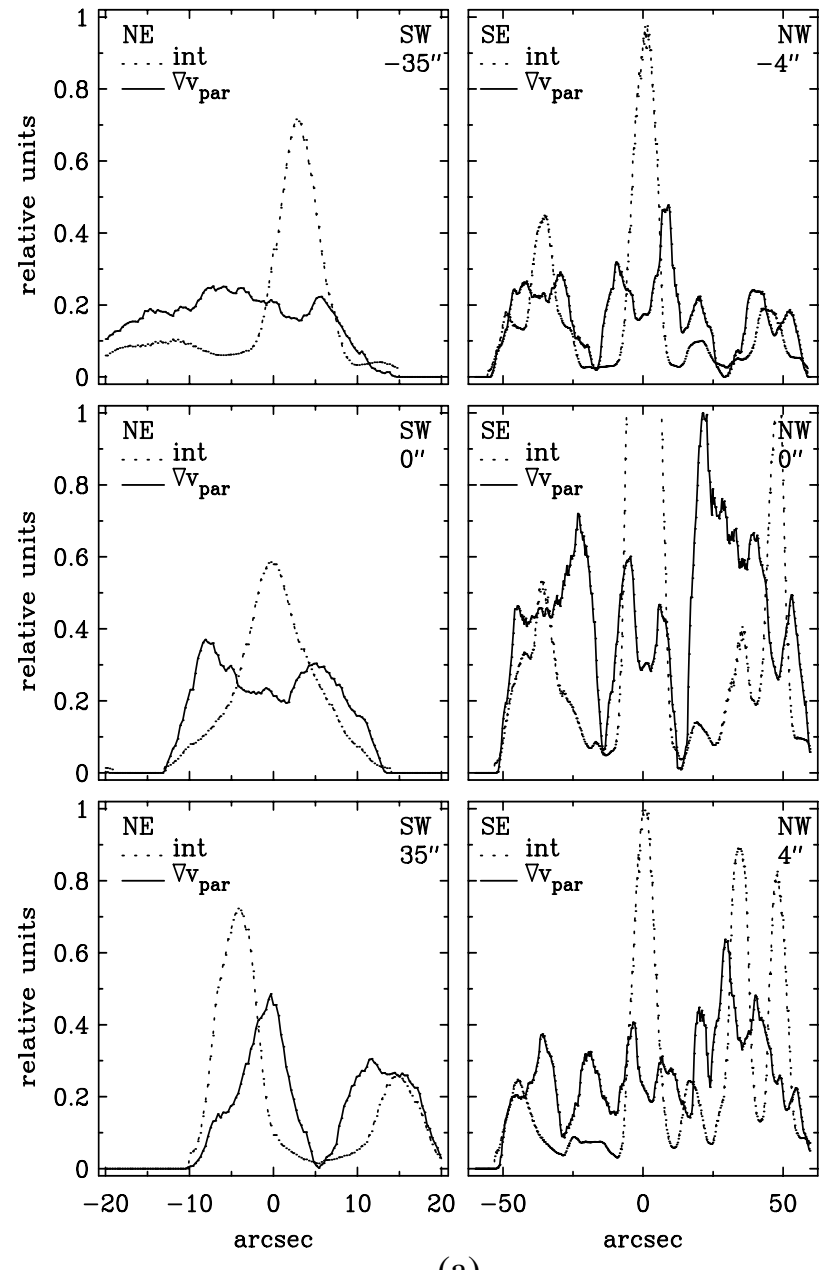

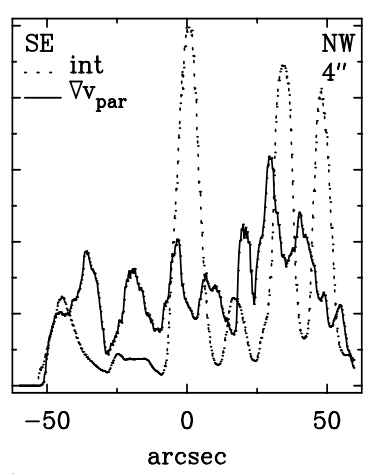

(a)

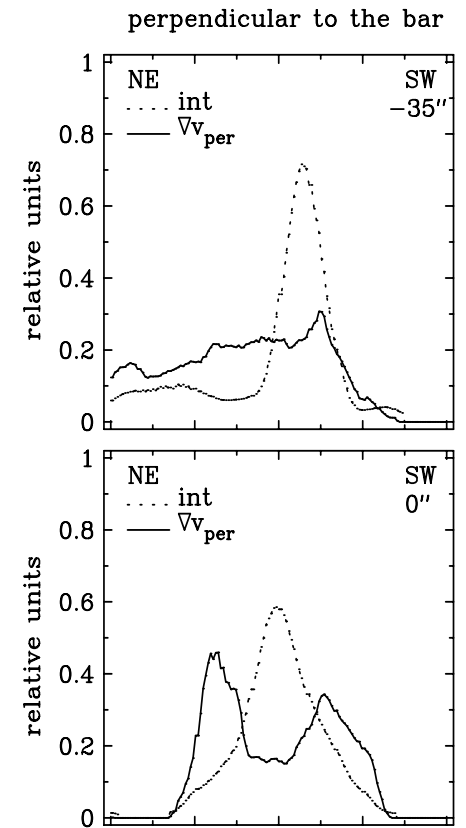

parallel to the bar
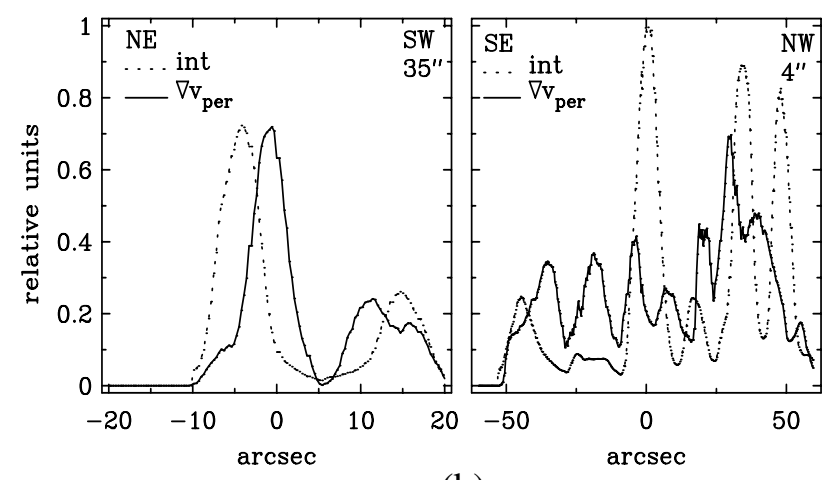

(b)
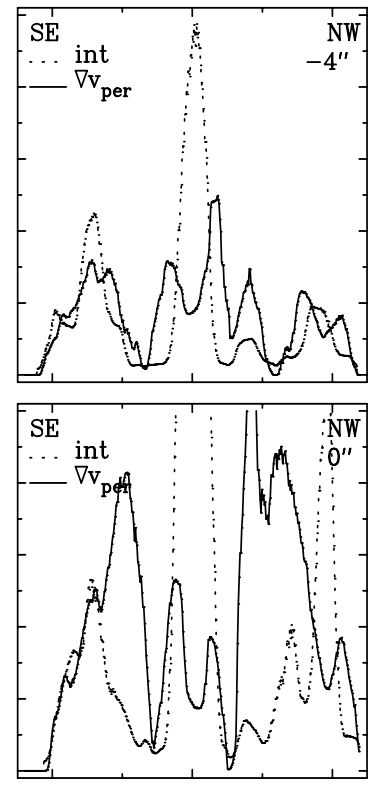

Fig. 13. a) Profiles of the normalized $\mathrm{H} \alpha$ surface brightness and residual velocity gradient parallel to the bar. The tracks of the profiles are the same as those in Fig. 12a. b) Same as a) but for the residual velocity gradient perpendicular to the bar. Note the offsets and anticorrelations of the intense star forming regions and regions of high velocity gradient shown in this figure and in Fig. 13a (see text).

angle of the galaxy, and assuming that the dust lanes in the observations from Pérez-Ramírez et al. (2000) denote the lines of flow, and that the spiral arms are trailing (this implies that the western part of the galaxy is closer to the observer than the eastern part). The resultant pattern of projected velocities is shown in Fig. 14d. Although the pattern is confined to the trajectory of the dust lanes, it agrees remarkably well with the projected residual velocity pattern in Fig.14c in almost all respects. The only differences are seen towards the $\mathrm{N}$ and $\mathrm{S}$ of the projected inner disc, where the model predicts higher residual velocities than those shown in Fig. 14d. We explain this as follows: in the radial range from $10^{\prime \prime}$ to $20^{\prime \prime}$ our best rotation curve, shown in Fig. 5, gives an apparent peak rising to some $300 \mathrm{~km} \mathrm{~s}^{-1}$. It is probable that the peak, and the curve in this range, show enhanced apparent rotational velocity because we are not able to eliminate non-circular components in this range. Only the inner disc emits here, and opening up the free angle further reduces the signal to noise ratio to unacceptably low values. If we were able to make a rational interpolation between the rising rotation curve within $10^{\prime \prime}$ radius, and the slowly falling outer curve beyond $25^{\prime \prime}$, we might have attempted a more accurate separation of circular velocity and non-circular residual velocity in the outer part of the inner disc. However such an interpolation would be so far from linearity that it would be little more than guesswork. Although the H I distribution does not have the gaps of our $\mathrm{H} \alpha$ image, the angular resolution in the $\mathrm{H}$ I rotation curve given in Regan et al. (1996) is no better than 13" so cannot supply the information we need. The projected model field in Fig. 14d gives such good agreement over most of the spiral flow lines that we feel secure in claiming that the discrepancies to the north and south of the inner disc are most probably due to the effects of rotation curve subtraction described here.

\subsection{A scenario for circumnuclear spirals}

A generic explanation for the presence of circumnuclear spirals was given by Englmaier \& Shlosman (2000). They showed that in a galaxy where there is a clearly identified ILR, the response of gas to forcing by a bar can give rise, for an appropriate central mass distribution and range of sound speed, to a spiral pattern in the gas which can be present all the way down to within tens of parsec of the nucleus. They predicted that the 

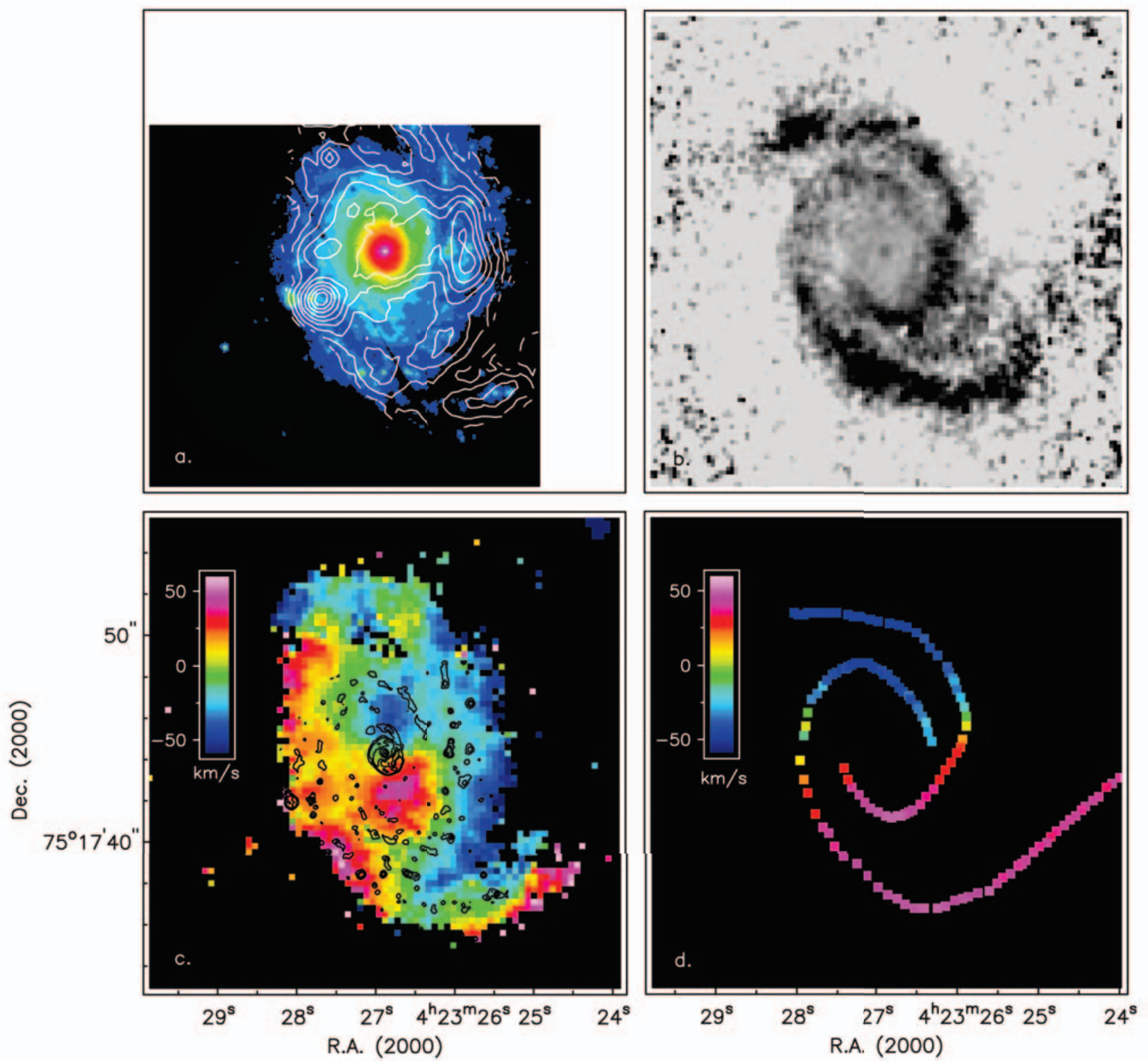

Fig. 14. a) $H S T$-NICMOS $H$ band image of the central part of NGC 1530 with $\mathrm{H} \alpha$ isointensity contours overlaid at $1^{\prime \prime}$ resolution. b) Grey-scale representations of the $J-K$ colour index image. Redder colours are indicated by the darker shades. Image taken from Fig. 2 of Pérez-Ramírez et al. (2000). c) Residual velocity map of 1 " resolution of the central part of NGC 1530. This map was obtained after subtracting the model velocity field obtained from the modified rotation curve in the center from the velocity map (see text details). Superposed are isointensity contours of the unsharp mask from the HST-NICMOS image shown in Fig. 15. d) Projected velocity field in the line of sight assuming a gas flow of constant amplitude $\left(60 \mathrm{~km} \mathrm{~s}^{-1}\right)$ along the observed dust lanes (Fig. 14b) in the inner part of NGC 1530. All images, a) to d), show the same area of the centre of NGC 1530.

arm/interarm contrast should be low, as found in near IR observations of these structures by Laine et al. (1999), and by PérezRamírez et al. (2000). The general framework for this kind of spiral structure does appear to be present in NGC 1530, since the form of the rotation curve has been shown by both Reynaud \& Downes (1997) and by Regan et al. (1996) to give rise to a predicted ILR. Reynaud \& Downes (1997) predict an outer ILR at $\sim 1.2 \mathrm{kpc}$ from the nucleus, and an inner ILR some $100 \mathrm{pc}$ from the nucleus. Here all we wish to assert is the consistency of our results with this type of models. We do not attempt to extend the work by any type of detailed modeling exercise.

\section{Discussion and concluding remarks}

\subsection{Three kinematic regimes}

NGC 1530 is a dramatically barred galaxy with very well defined morphological features, so it is not surprising that there is considerable recent work in the literature on its gas kinematics. However, the use of an $\mathrm{H} \alpha$ emission line map at $\sim 1^{\prime \prime}$ resolution over the major part of the disc has enabled us to probe more deeply some of its kinematical properties. We have obtained a very well resolved rotation curve which is symmetric for the two independent halves of the galaxy. It was easy to 


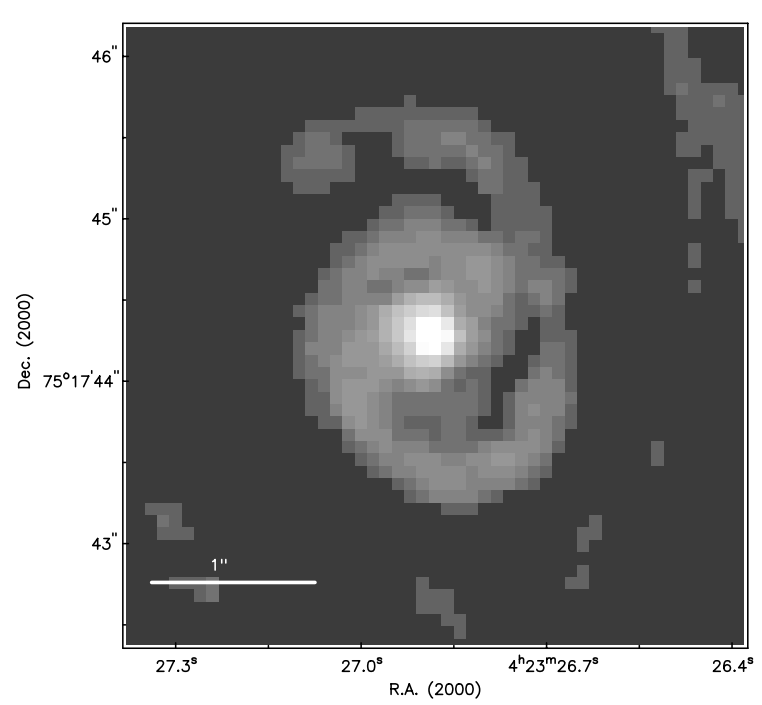

Fig. 15. Unsharp masked NICMOS image (Fig. 14a) of the central part of NGC 1530.

detect the presence of non-circular components of the internal motion, in three dynamically distinct zones: streaming motions in the spiral arms, highly elliptical orbits around the bar, and an inwardly spiralling component particularly marked in the circumnuclear disc within $2 \mathrm{kpc}$ of the centre. By the conventional technique of subtracting off a two-dimensional projected model rotation curve from the observed velocity field we produced a map of these non-circular residual velocities, which include components of amplitude well over $100 \mathrm{~km} \mathrm{~s}^{-1}$ in the bar-induced elliptical orbits. The fact that the different kinematical regimes referred to above occur in distinct ranges of galactocentric radius, facilitated the separation of the circular and the radial components. However both in the range of the spiral arms, from $63^{\prime \prime}$ to $90^{\prime \prime}$ from the centre, and in the inner disc, out to some $5^{\prime \prime}$ from the centre, we used auxiliary dynamical reasoning to effect an improved separation. We assumed that the departures from smooth radial dependence found in these ranges cannot be due to rapidly radially varying mass distributions, since no such variations are present in near-IR broad band images which trace the stellar mass, and must instead be due to projected components of local non-circular motions not well separated out from the true rotation curve. Based on this, we interpolated smooth underlying envelopes to the two sections of the observed rotation curve, and used this smoothed version of the rotation curve to produce revised maps of the residual velocity field, in which the streaming motions in the arms and the spiralling motions towards the centre in the circumnuclear disc are better represented. These procedures do not affect the strong residual velocities around the bar, which occur in an intermediate range of radii, but do yield a more accurate overall map of the non-circular velocity field.

\subsection{The key role of velocity gradients}

By far the most notable new results in this paper refer to the gradients in velocity which we have been able to detect directly and measure. Particularly striking is the map of velocity gradients perpendicular to the bar, in the range of the bar (Fig. 11d). Although in previous work on this and other barred galaxies the presence of a rapid jump in radial velocities, from $\sim+100 \mathrm{~km} \mathrm{~s}^{-1}$ to $\sim-100 \mathrm{~km} \mathrm{~s}^{-1}$ as one transits the bar, has been detected, this is the first case where the relevant field has been closely mapped. It is essentially a shear field, since when we compare this with the velocity gradient map in the direction along the bar, (i.e. along the direction of the gas flow vectors) the perpendicular gradient is considerably stronger. We do note that a perfect separation into components parallel to and perpendicular to the flow is not possible even in principle in observations purely of radial velocities, but that the dynamics of the situation, with the $x_{1}$ orbits around the outer lengths of the bar, is very favourable for a rather good practical separation in NGC 1530. It is notable that the zones of strongest gradient coincide precisely with the dust lanes even in fine detail. We have detected gradients as steep as $0.35 \mathrm{~km} \mathrm{~s}^{-1} \mathrm{pc}^{-1}$ in a direction which is essentially perpendicular to the flow vector along the bar, i.e. these are shear gradients. Comparing this map with the $\mathrm{H} \alpha$ integrated intensity map in Fig. 11a we can see that there is a complete anticorrelation between high values of shear gradient and the local star formation rate. Massive stars are not being formed in zones of high shear. This is not a surprising result at all. The work of Reynaud \& Downes (1998) was directed exactly at this point in NGC 1530, and came to this conclusion.

\subsection{Parallel shocks inhibit star formation; perpendicular shocks stimulate it}

The only advance we claim here is to have completed and detailed maps of the phenomenology, with measured velocities and velocity gradients, both in amplitude and direction. From these we can extract several interesting semi-quantitative conclusions. Firstly, although star formation regions avoid strong shear, they do not necessarily avoid high non-circular velocity. Specifically there is a star forming region, $20^{\prime \prime}$ to the NW of the galactic centre, in a region of high velocity along the bar (greater than $100 \mathrm{~km} \mathrm{~s}^{-1}$ in a negative sense, deep blue in the residual velocity map). This region is, however, in a trough of velocity gradient: less than $0.05 \mathrm{~km} \mathrm{~s}^{-1} \mathrm{pc}^{-1}$ both parallel to and perpendicular to the bar. It is interesting to note that this $\mathrm{H}$ II region lies along the trailing edge of the bar, and that this edge is the site of a number of regions, while the leading edge of the bar does not show any strong regions. This is not a common situation and could lead to a conclusion which questions the placing of corotation near the end of the bar. However, we do not believe that this is necessary. The bar is so strong that the spray effect illustrated in Fig. 8, should stimulate star formation along the trailing edge at positions of the arrow tips, where gas on quasi-elliptical orbits collides with gas in the bar as it begins to reverse its direction. Another way to consider this, is that we may be observing effects of a 4:1 ultraharmonic resonance, but highly flattened by the strong bar. All the other major star forming regions in the central disc, along the bar, at the ends of the bar, and in the arms, occur in regions of low residual velocity as well as low velocity gradient. 
Secondly, there does appear to be a relationship between shocks (as picked out by steep velocity gradients along the direction of the gas flow) and star forming zones. As seen particularly well in the map of velocity gradients parallel to the bar (Fig. 11c), linear zones of high velocity gradient along the bar, at the edges of the central disc, and even in the spiral arms (not shown in the cited figure) tend to accompany the regions of strongest star formation, with the zone of steep gradient offset from the centre of the nearby star forming region by a distance of a few hundred parsecs. This phenomenon could have two causes: either the compression along the line of flow is causing major gas condensation which leads to star formation, or outflow from star forming regions is impinging on the general flow to yield high gradients along the flow direction. We have considerable evidence from detailed kinematical studies of individual luminous H II regions in a number of galaxies (Relaño et al. 2003a,b) that outflow at velocities of between $50 \mathrm{~km} \mathrm{~s}^{-1}$ and $100 \mathrm{~km} \mathrm{~s}^{-1}$ is occurring in most of them, so the second phenomenon could well occur. However, the scales of some of the gradients seen in Fig. 11c and the fact that in some cases these occur offset to one side of a star forming region and not to the other indicate that pre-stellar compression shocks are also being observed here. Quantification and modelling are clearly beyond the scope of the present paper, but these data may well offer a possible valuable route to the specification of star formation conditions. It is, of course, the difficulties of pinning down observationally the conditions prior to star formation rather than any lack of theoretical offer which limits our understanding of the phenomenon.

\subsection{Quantified spiral inflow towards the nucleus}

A two-dimensional map of the non-circular velocities also gives us the chance to follow kinematically the gas flow which is picked out morphologically by the dust lanes. Although projection effects are quite severe on observations of a flow whose vector in the plane of the galaxy is swinging through more than $270^{\circ}$, we have prima facie evidence that we are able to follow this flow down to within $300 \mathrm{pc}$ of the centre in this galaxy. This is not very close to the nucleus, but bearing in mind that NGC 1530 is $37 \mathrm{Mpc}$ from us we could certainly expect interesting results from this method for closer objects.

Acknowledgements. We owe thanks to Dr. M. Regan for kindly providing us his $\mathrm{H} \alpha$ rotation curve data, Dr. D. Pérez-Ramírez for help with observations and Dr. S. Laine for directing our attention to the material in Figs. 15 and 14a and for discussions on its intepretation. We thank AURA for the use of the image of NGC 1530 in Fig. 9 b. We are happy to thank Dr. Denis Downes for his fast and efficient refereeing, as well as for his encouraging remarks. The WHT is operated on the island of La Palma by the Isaac Newton Group in the Spanish Observatorio del Roque de los Muchachos of the Instituto de Astrofísica de Canarias. Based on observations with the NASA/ESA Hubble Space Telescope, obtained from the data Archive at the Space Telescope Science Institute, which is operated by the Association of Universities for Research in Astronomy, Inc., under NASA contract NAS 5-26555. This work was supported by the Spanish DGES (Dirección General de Enseñanza Superior) via Grants PB910525, PB94-1107 and PB97-0219 and by the Ministry of Science and Technology via Grant AYA2001-043.

\section{References}

Athanassoula, E. 1984, Phys. Rep., 114, 319

Athanassoula, E. 1992, MNRAS, 259, 345

Begeman, K. 1989, A\&A, 223, 47

Buta, R. 1984, Ph.D. Thesis University of Texas, Austin

Buta, R., Block, D. L., \& Knapen, J. H. 2003, AJ, in press

de Vaucouleurs, G., de Vaucouleurs, A., Corwin, H. G., et al. 1991, Third Reference Catalogue of Bright Galaxies (RC3) (New York: Springer)

Downes, D., Reynaud, D. Solomon, P. M., \& Radford, S. J. E. 1996, ApJ, 461, 186

Englmaier, P., \& Shlosman, I. 2000, ApJ, 528, 677

Elmegreen, B. G., Palous, J., \& Ehlerova, S. 2002, MNRAS, 334, 693

Erwin, P., \& Sparke, L. S. 2002, AJ, 124, 65

Freedman, W. L., Madore, B. F., Gibson, B. K., et al. 2001, ApJ, 553, 47

Greve, A., Reynaud, D., \& Downes, D. 1999, A\&A, 348, 394

Huntley, J. M., Sanders, R. H., \& Roberts, W. W. Jr. 1978, ApJ, 221, 521

Jenkins, A. A., \& Binney, J. 1994, MNRAS, 270, 703

Kennicutt, J. R. 1989, ApJ, 344, 685

Knapen, J. H. 1998, MNRAS, 297, 255

Lindblad, P. O., \& Jorsater, S. 1981, A\&A, 97, 56L

Laine, S., Knapen, J. H., Pérez-Ramírez, D., Doyon, R., \& Nadeau, D. 1999, MNRAS, 302, L3

Pérez-Ramírez, D., Knapen, J. H., Peletier, R. F., et al. 2000, MNRAS, 317,234

Relaño, M., Beckman, J. E., Rozas, M., \& Zurita, A. 2003a, Rev. Mex. Astron. Astrofis., 15, 205

Relaño, M., et al. 2003b, in preparation

Reynaud, D., \& Downes, D. 1997, A\&A, 319, 737

Reynaud, D., \& Downes, D. 1998, A\&A, 337, 671

Reynaud, D., \& Downes, D. 1999, A\&A, 347, 37

Regan, M., Teuben, P., Vogel, S., \& van der Hulst, T. 1996, AJ, 112, 2549

Regan, M., Vogel, S., \& Teuben, P. 1995, ApJ, 449, 576

Regan, M., Vogel, S., \& Teuben, P. 1997, ApJ, 482, L143

Roberts, W. W. Jr., Huntley, J. M., \& van Albada, G. D. 1979, ApJ, 233, 67

Rozas, M., Sabalisck, N., Beckman, J. E., \& Knapen, J. H. 1998, A\&A, 338, 15

Rozas, M., Zurita, A., Beckman, J. E., \& Pérez, D. 2000, A\&AS, 142, 259

Toomre, A. 1964, ApJ, 139, 121

Tully, R.B., Fisher, J.R. 1988, Catalog of Nearby Galaxies (Cambridge University Press)

Shlosman, I., Frank, J., \& Begelman, M. C. 1989, Nature, 338, 45

Young, J. S., Xie, S., Kenney, J. D. P., \& Rice, W. L. 1989, ApJS, 70, 699

van der Kruit, P., \& Shostak, G. S. 1982, A\&A, 105, 351

Wray, J. D. 1988, The Color Atlas of Galaxies (Cambridge University Press) 